\title{
Experimental and kinetic modeling study of the shock tube ignition of a large oxygenated fuel: Tri-propylene glycol mono-methyl ether
}

\author{
Ultan Burke ${ }^{1}$, William J. Pitz ${ }^{2}$, Henry J. Curran ${ }^{1}$ \\ Corresponding author: $U$. Burke \\ RWTH Aachen University \\ Schinkelstraße 8 \\ 52062 Aachen | GERMANY \\ Email: burke@pcfc.rwth-aachen.de
}

${ }^{2}$ Lawrence Livermore National Laboratory, 7000 East Avenue, Livermore, California, USA

${ }^{1}$ Combustion Chemistry Centre, School of Chemistry, NUI Galway, Ireland.

\begin{abstract}
Tri-propylene glycol monomethyl ether (TPGME) is an important oxygenated fuel additive that can be used to reduce soot in diesel engines. However, a validated chemical kinetic model that incorporates the low- to high-temperature chemistry, needed to simulate ignition in a diesel engine is not available for TPGME. In addition, no fundamental experimental data are available that can be used to validate a TPGME mechanism. In this study, a surrogate chemical kinetic model for TPGME that includes low- and high-temperature chemistry has been developed, and shock tube ignition delay time data has been acquired for its validation at $0.25 \%$ TPGME for temperatures in the range of $980-1545 \mathrm{~K}$, at pressures of 10 and $20 \mathrm{~atm}$, and at equivalence ratios of $\varphi=0.5,1.0$ and 2.0. The predictions from the model have been compared to the experimental measurements with good agreement. Under the experimental conditions investigated in the shock tube, TPGME was found to be consumed by molecular elimination reactions and also $\mathrm{H}$-atom abstraction by $\dot{\mathrm{H}}$ atoms and $\dot{\mathrm{O}} \mathrm{H}$ and $\mathrm{HO}_{2}$ radicals. In performing sensitivity analyses it was found that the ignition of TPGME is most sensitive to reactions involving propene. Considering how the sensitivity analyses change with pressure, the most
\end{abstract}


sensitive reactions involved $\dot{\mathrm{H}}$ atoms at $10 \mathrm{~atm}$ and $\mathrm{HO}_{2}$ radicals at $20 \mathrm{~atm}$. With respect to the effect of equivalence ratio, reactions involving $\dot{H}$ atoms are relatively more sensitive under fuelrich conditions while those involving $\mathrm{HO}_{2}$ radicals are relatively more sensitive under fuel-lean conditions. Further experimental work is needed to enable validation of the model under lowtemperature conditions. TPGME was compared to $n$-heptane which has similar ignition properties based on Cetane Number. Predictions showed that TPGME has a higher overall reactivity compared to $n$-heptane. In addition, TPGME is shown to produce significantly less soot precursor species when TPGME predictions are compared to $n$-heptane.

Keywords: TPGME, oxygenated fuel, chemical kinetic modeling, shock-tube ignition 


\section{Introduction}

Tri-propylene glycol monomethyl ether (TPGME) is an attractive oxygenated additive that is effective in reducing soot emissions in diesel engines [1-3]. Gonzalez et al. [2] found TPGME and dibutylmaleate (DBM) were the most promising oxygenates out of 8 oxygenates tested for addition to a low-sulfur diesel fuel for reduction of particulate emissions. They determined that adding $20 \%$ by volume of TPGME to diesel fuel reduced particulate emissions in a diesel engine by $47 \%$. Investigating these two most promising oxygenate additives, Mueller et al. [1] found that TPGME was more effective in reducing soot than DBM for all test conditions investigated in an optical diesel engine. They investigated 20\% TPGME and 80\% 2,2,4,4,6,8,8heptamethylnonane (a diesel reference fuel) as an oxygenated "diesel" fuel mixture. Kinetic model calculations in their study showed that $30 \%$ of the oxygen contained in DBM is unavailable for reducing soot precursors whereas all of the oxygen in TPGME is available. In a constant volume combustion chamber that mimics conditions in a modern diesel engine, Manin et al. [3] found that a 50/50 mixture of TPGME and n-hexadecane (a diesel primary reference fuel) gave lower sooting levels than a biodiesel methyl ester surrogate composed of neat methyldecanoate. They attributed this difference in sooting levels to "chemical structural differences on how the oxygen is bonded in the two oxygenated molecules".

TPGME has a low toxicity and vapor pressure and is currently used practically as an industrial solvent, chemical intermediate, and in inks for ballpoint or felt-tipped pens [4]. Commercially, TPGME is produced only as a mixture of eight isomers, Fig. 1. 


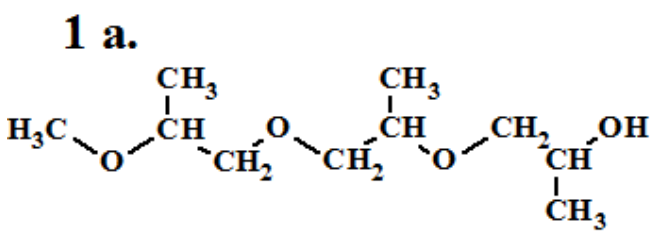

2 a.

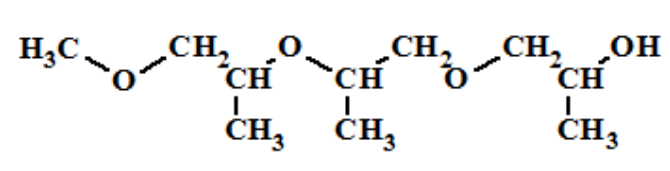

1 b.

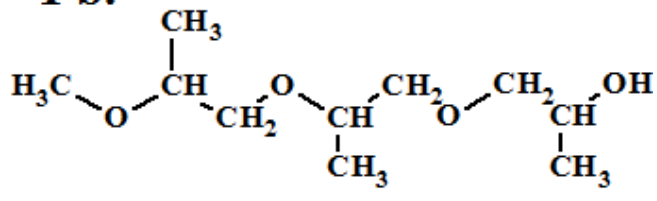

2 b.

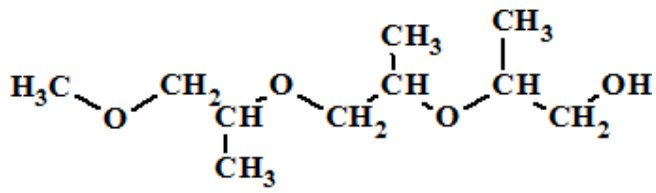

2 c.<smiles>CO[C@H](C)COCC(C)O[C@H](C)CO</smiles><smiles>COC(C)COC(C)COC(C)CO</smiles>

4 a.<smiles>COCC(C)OC(C)COC(C)CO</smiles>

4 b.<smiles>COC[C@@H](C)OC[C@H](C)OC(C)CO</smiles>

Figure 1: Structural isomers present in the isomeric mixture studied ranked in 4 groups according to their expected reactivity. (Group 1 has the highest expected reactivity.)

Propylene glycol ethers in general are manufactured in closed reactors using propylene oxide and methanol as precursors. In order to produce high yields of TPGME, high pressure, temperature and molar concentrations of propylene oxide and methanol are required and the eventual isomeric mixture of TPGME is isolated via distillation $[4,5]$. The isomers of the mixture have similar boiling points $\left(\sim 243{ }^{\circ} \mathrm{C}\right)$ and thus it was impractical to separate and quantify them; therefore to simplify modeling of the mixture, one isomer was chosen as a surrogate to represent 
the eight-isomer mixture. Isomer 3a (Fig. 1 and 2) was chosen as a surrogate for the mixture because its high temperature mechanism has been previously developed and was available [1]. If the isomers are ranked into 4 reactivity groups (Fig. 1), it is expected to be in the third group based on reactivity arguments given in the Reaction Rate Constant Section 3.1.

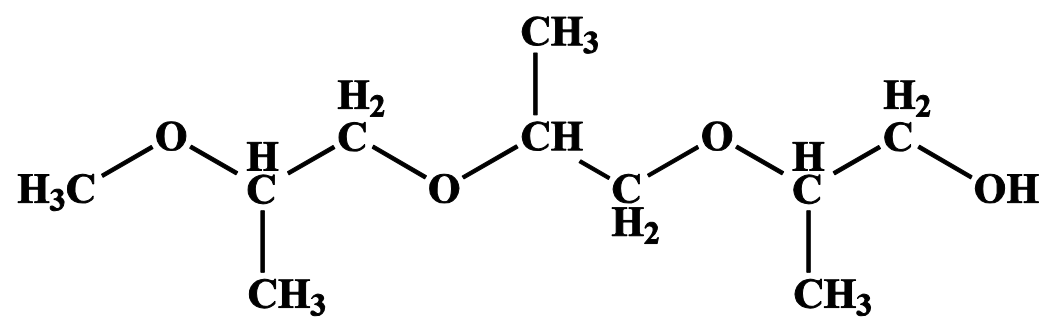

Figure 2: Isomer 3 a chosen to represent TPGME mixture for model development.

TPGME has been used in research diesel engines yielding near non-sooting combustion. This effect has motivated an interest in the mechanism of the oxidation of TPGME. A surrogate chemical kinetic mechanism for TPGME will help us understand and optimize its combustion in engines. However, a TPGME mechanism that is valid at high pressures and over the low- to high-temperature range associated with combustion in engines is not available. In order to develop a model, fundamental experimental data is also required in order to provide validation targets which have not previously been available.

From a molecular-structure point of view, TPGME is interesting. It is highly branched with four oxygen atoms distributed throughout the molecule, Fig. 1. It contains both the alcoholic and ether moiety, with secondary $\mathrm{C}-\mathrm{H}$ bonds adjacent to the alcoholic functional group and primary, secondary and tertiary $\mathrm{C}-\mathrm{H}$ bonds adjacent to the ether moieties distributed throughout the molecule. Most other oxygenated fuels investigated previously in the literature (methanol [6], iso-butanol [7], dimethyl ether [8,9] and methyl tert-butyl ether [10] etc.) are much smaller than TPGME in molecular size and are either lightly-branched or straight-chained in their skeletal 
structure. These characteristics of TPGME represent a challenge for chemical kinetic model development.

A chemical kinetic model for TPGME has been developed previously [1, 11] to address its hightemperature chemistry and production of soot precursor species under conditions in diesel engines (high pressures $99 \mathrm{~atm}$ and low temperatures $767 \mathrm{~K}$ ), and this model also took the surrogate approach, by only considering the isomer shown in Fig. 2. Westbrook et al. [11] presented comparisons of a range of oxygenated fuel additives (methanol, ethanol, dimethyl ether, dimethoxy methane, methyl butanoate, TPGME, dimethylcarbonate and DBM) and their effect on the formation of soot precursor species (ethylene, acetylene, propyne, etc.). It was found that the formation of these species was somewhat reliant on the percentage oxygen by weight in the overall fuel mixture ( $n$-heptane + oxygenated additive in "air"). Also, it was noted that the even distribution of O-atoms throughout the TPGME molecule meant that its soot reduction capabilities were quite efficient, preventing the $\mathrm{C}$-atoms in the molecule from proceeding to form soot precursor species. Due to a lack of fundamental data, no validation targets were available for this model, and its predictions were purely theoretical. Although this model may have been adequate in predicting the production of soot precursor species and in predicting ignition when TPGME was present in modest concentrations (perhaps up to 5\%) in a diesel surrogate-fuel mixture, the mechanism did not contain the low-temperature reactions necessary to predict ignition when TPGME was present in high concentrations $(15-50 \%$ by volume in Manin et al. [3]).

The present work was undertaken to develop a chemical kinetic model for TPGME that is capable of predicting the chemistry at low- to high-temperatures and for pressures encountered in a diesel engine. Additionally, it was not possible to validate the previous TPGME model since no 
fundamental experimental data were available. The additional goal of this work was to acquire fundamental shock tube ignition data in order to validate the TPGME mechanism. These data combined with the model represent the first validated chemical kinetic mechanism available for the evaluation of TPGME as a fuel or fuel additive. The inclusion of low-temperature reaction pathways for the oxidation of TPGME is important because it allows the model to be used to probe ignition under conditions found in diesel engines. Once the model has been developed, it can be reduced in size and used in multi-dimensional engine codes to simulate the combustion of TPGME/diesel fuel mixtures and to understand the mechanism of TPGME in reducing particulate emissions from diesel engines. The inclusion of these low-temperature oxidation pathways is also important in discerning whether or not these pathways play a role in the prediction of the newly measured experimental data presented in this study.

\section{Experimental Description}

Ignition delay times were measured using a heated high-pressure shock tube described in detail previously [12]. Briefly, it is cylindrical with a driven section of $5.7 \mathrm{~m}$ and a driver section of 3.0 $\mathrm{m}$, with an internal diameter of $63.5 \mathrm{~mm}$. The full list of mixtures studied and their compositions are provided in Table 1 , where $p_{5}$ is the nominal pressure behind the reflected shock wave. The exact conditions for each shock tube experiment and the measured ignition times are provided as Supplementary Material.

Table 1: Experimental conditions investigated in shock tube experiments

\begin{tabular}{ccccccc}
\hline Mix No. & $\boldsymbol{p}_{\mathbf{5}} / \mathbf{~ a t m}$ & $\boldsymbol{\varphi}$ & TPGME / Mole \% & $\mathbf{O}_{\mathbf{2}} /$ Mole $\%$ & Ar / Mole \% & $\boldsymbol{T}_{\mathbf{5}} / \mathbf{K}$ \\
\hline \hline 1 & 10 & 0.50 & 0.25 & 6.75 & 93.00 & $988-1316$ \\
2 & 10 & 1.00 & 0.25 & 3.38 & 96.38 & $1154-1537$ \\
3 & 10 & 2.00 & 0.25 & 1.69 & 98.06 & $1222-1501$ \\
4 & 20 & 0.50 & 0.25 & 6.75 & 93.00 & $1043-1334$ \\
5 & 20 & 1.00 & 0.25 & 3.38 & 96.38 & $1089-1406$ \\
6 & 20 & 2.00 & 0.25 & 1.69 & 98.06 & $1187-1491$ \\
\hline \hline
\end{tabular}


Mixtures were prepared manometrically in a stainless steel mixing vessel. A magnetically controlled fan was used to ensure homogeneity within the mixtures. All mixtures were allowed to mix for at least one hour before starting experiments. TPGME was obtained from SigmaAldrich at a purity of $>97.0 \%$ and as mentioned above, it is a mixture containing 8 isomers, Fig. 1. The sample was treated to remove any peroxides by elution through alumina (80-200 mesh, Fisher Chemical A540, conditioned overnight at $673 \mathrm{~K})$. Oxygen, argon and driver gases, helium and nitrogen, were obtained from BOC Ireland at purities of $\geq 99.50 \%$ for Ar, and $\geq 99.99 \%$ for the others, and were used without further purification.

TPGME has a very low vapor pressure $(0.80 \mathrm{~Pa}$ at $298 \mathrm{~K})$ and high boiling point $(516 \mathrm{~K})$, and the shock tube, mixing vessel and manifold were heated to $423 \mathrm{~K}$ during experiments to prevent condensation. It is also noted that $423 \mathrm{~K}$ is below TPGME's reported auto-ignition temperature of $550 \mathrm{~K}$ [4]. Laser absorption measurements were employed in order to ensure that no condensation was occurring (see Fig. 3), this method was also employed and described by Mével et al. [13] and Nakamura et al. [12]. Briefly, the absorption presented in Fig. 3 is the $\mathrm{C}-\mathrm{H}$ bond absorption measured using a helium-neon laser at $3.39 \mu \mathrm{m}$. Two Thorlabs PDA20H-EC photodiode array detectors were used to measure the absorption; one was positioned before the test-cell and a second after it in order to provide both a reference and measured signal. Using the gaseous pressure in the test-cell the fuel concentration could be calculated. This method was first performed in a test-cell setup and the measurements used to create a calibration curve. Thereafter, measurements were taken in-situ confirming the expected concentration of TPGME in the experiments. Further details on this method have been provided by Mével et al. [13] and Nakamura et al. [12]. 


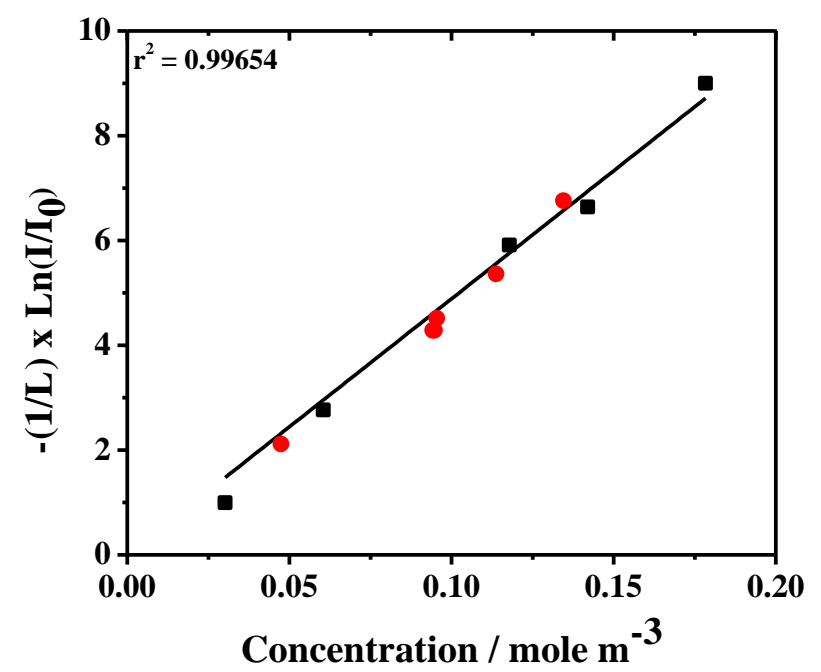

Figure 3: Laser Absorption Measurements. Test cell $=\mathbf{m}$. In-situ $=\bullet$. Line $=$ linear correlation to test cell measurements. Absorption of $\mathrm{C}-\mathrm{H}$ bonds, measured at a wavelength of $3.39 \mu \mathrm{m}$, using a He-Ne laser.

The measured ignition delay times are defined as the time difference between the arrival of the reflected shock wave at the side wall transducer to the maximum rate of pressure increase due to ignition, an example of which is shown in Fig. 4. Depicted is the experimentally measured pressure (solid black line), the idealized constant pressure after passage of the reflected shock wave (dashed black line) and the method of determining the ignition delay time from the pressure trace as described above (solid and dashed red lines). The accurately measureable range of ignition delay times in this facility is $50-5000 \mu$ s. Shock attenuation was measured to be $2-3$ $\% / \mathrm{m}$. Negligible pressure rise between arrival of the reflected shock wave and ignition was noted (Fig. 4). 


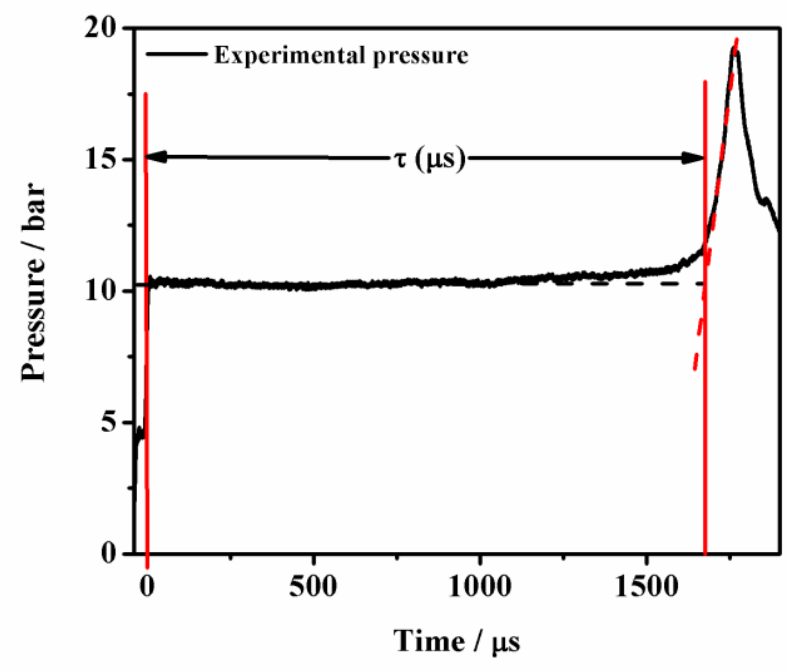

Figure 4: Experimental pressure trace. Reflected shock conditions: $p=10.4 \pm 0.1 \mathrm{~atm}, T=1154$

$$
\pm 10 \mathrm{~K}, \phi=1.0 \text {. }
$$

Experimental uncertainty was estimated as $\pm 15 \%$ in ignition delay time. This includes contributions from the mixture composition, initial pressure and temperature measurements, and the shock velocity calculation. Post-shock conditions were computed using the Gaseq code [14]. 


\section{Chemical Kinetic Model Formulation}

\subsection{Reaction Rate Constants}

A TPGME mechanism has been developed that includes low-temperature chemistry for the isomer shown in Fig. 2. This isomer has been used as a surrogate to represent all of the isomers in the isomeric mixture obtained from Sigma-Aldrich. To update the mechanism, we first revised the $\mathrm{C}_{1}$ to $\mathrm{C}_{4}$ chemistry and the high-temperature reactions of TPGME. The $\mathrm{C}_{1}$ to $\mathrm{C}_{4}$ chemistry has been adopted from AramcoMech1.3 [15]. The high-temperature reactions of TPGME have also been updated from a previous TPGME mechanism [11]. For the initial fuel decomposition reactions, the rate constants for the four-centered molecular elimination reactions in TPGME were based on a similar reaction in methyl tert-butyl ether (MTBE). The rate constant for MTBE was taken directly from the $a b$ initio calculations of Chen and Bozzelli [16] and corrected for reaction degeneracy. A comparison of this rate constant with other rate constants for MTBE molecular elimination from the literature is shown in Fig. 5. 


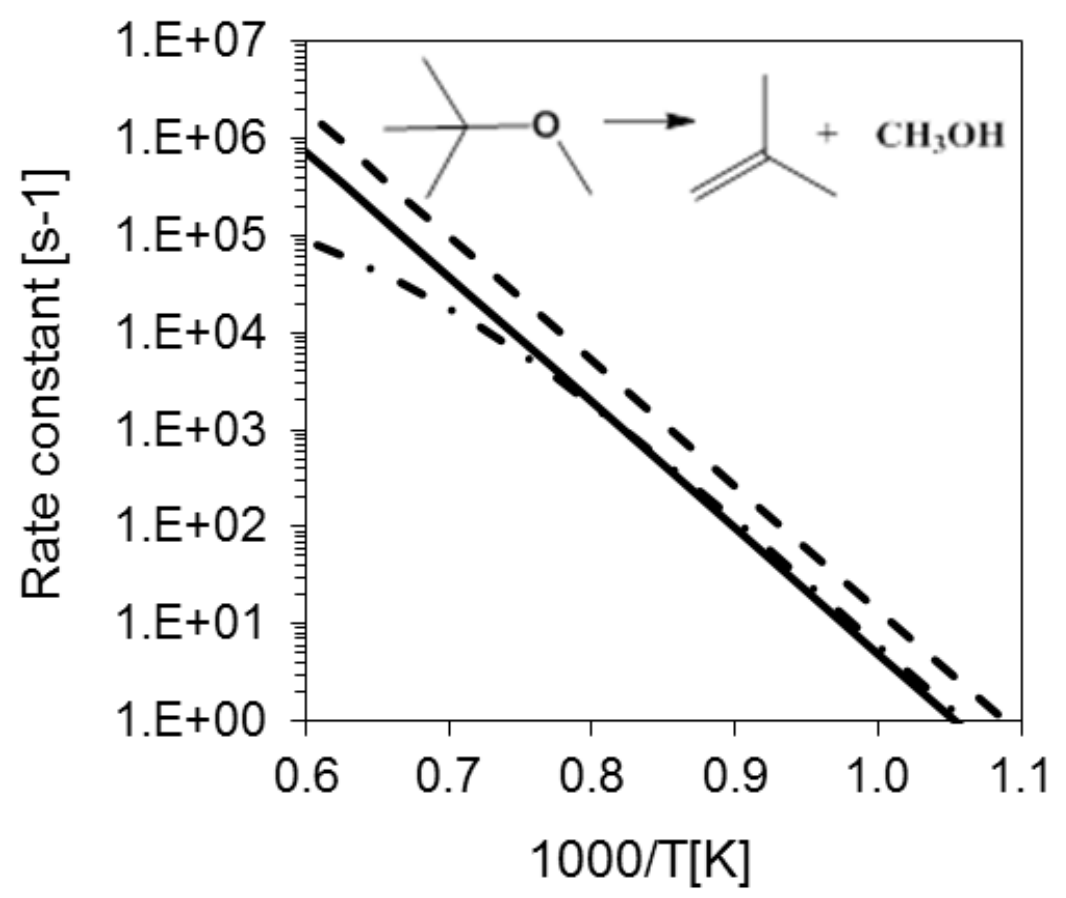

Figure 5: Rate constants for the 4-centered elimination reaction for MTBE. Solid line is from Brocard et al. [17], Dot-dash line is from Yasunaga et al. [10] and the dashed curve is from Chen and Bozzelli [16].

The Yasunaga et al. [10] rate constant plotted in Fig. 5 includes fall-off but because of the large number of additional vibration modes in TPGME compared to MTBE, TPGME is not expected to exhibit significant fall-off for the temperature and pressure range of the this study. The uncertainty in the MTBE elimination rate is estimated to be a factor of 3. In MTBE, the molecular elimination reactions only involve primary $\mathrm{C}-\mathrm{H}$ bonds. In TPGME, these eliminations involve both primary and secondary $\mathrm{C}-\mathrm{H}$ bonds. Since the analogous molecular elimination rates in TPGME has not been specifically calculated and they involve both primary and secondary C$\mathrm{H}$ bonds, the uncertainty in its rate constant is estimated to be a factor of 10 . The four-centered elimination of water involving the alcohol group in TPGME was not included because the rate 
constant is expected to be about two orders of magnitude below that of eliminations involving primary or secondary $\mathrm{C}-\mathrm{H}$ bonds based on the water elimination rate constant measured by Rosado-Reyes et al. [18] for iso-butanol.

For H-atom abstraction reactions from TPGME, the abstraction rate constants from site 1 (Fig. 6) were taken by analogy to dimethyl ether [19-21]. For abstraction rate constants at sites that were adjacent to O-atoms, the rate constants at sites 5, 9, and 13 (Fig. 6) were assigned by analogy with 1-butanol and sites 3, 7, and 11 by analogy to 2-butanol. These rate constants were taken directly from Sarathy et al. [7]. The remaining abstractions sites involving methyl groups were adopted from Sarathy et al. [22]. Specific assignments of rate constants for other hightemperature reactions are described in the well-annotated reaction mechanism that has been provided as Supplementary data.

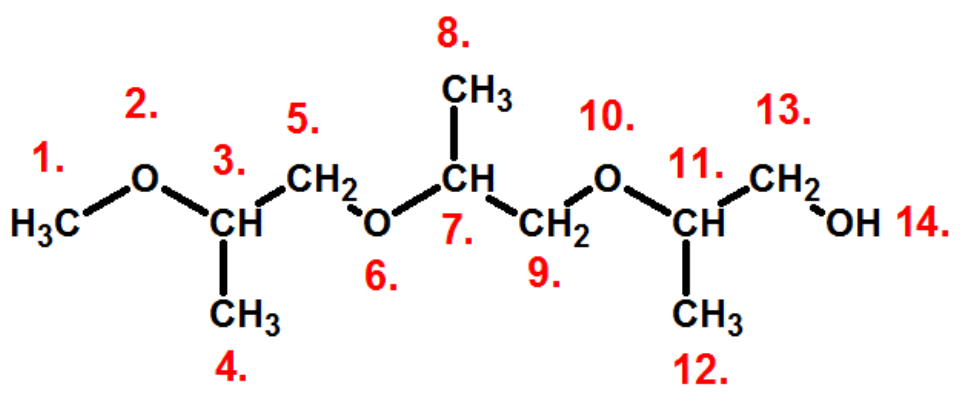

Figure 6: Surrogate TPGME isomer (3a, Fig. 1) with labeled sites denoting naming scheme of TPGME species.

The low-temperature reactions of TPGME have been added to the mechanism using the reaction classes and rules drawn from the alkane and butanol papers of Sarathy et al. [7, 22]. The following reaction classes have been included, following the numbering in [22] and in the accompanying mechanism available as Supplementary data:

\section{Low-Temperature Reaction Classes}

11. Addition of $\mathrm{O}_{2}$ to alkyl radicals $\left(\mathrm{R}+\mathrm{O}_{2}=\mathrm{RO}_{2}\right)$ 
12. $\mathrm{R}+\mathrm{RO}_{2}=\mathrm{RO}+\mathrm{RO}$

13. $\mathrm{R}+\mathrm{HO}_{2}=\mathrm{RO}+\mathrm{OH}$

14. $\mathrm{R}+\mathrm{CH}_{3} \mathrm{O}_{2}=\mathrm{RO}+\mathrm{CH}_{3} \mathrm{O}$

15. Alkyl peroxy radical isomerization $\left(\mathrm{RO}_{2}=\mathrm{QOOH}\right)$

16. Concerted eliminations $\left(\mathrm{RO}_{2}=\right.$ alkene $\left.+\mathrm{HO}_{2}\right)$

17. $\mathrm{RO}_{2}+\mathrm{HO}_{2}=\mathrm{ROOH}+\mathrm{OH}$

18. $\mathrm{RO}_{2}+\mathrm{H}_{2} \mathrm{O}_{2}=\mathrm{ROOH}+\mathrm{HO}_{2}$

19. $\mathrm{RO}_{2}+\mathrm{CH}_{3} \mathrm{O}_{2}=\mathrm{RO}+\mathrm{CH}_{3} \mathrm{O}+\mathrm{O}_{2}$

20. $\mathrm{RO}_{2}+\mathrm{RO}_{2}=\mathrm{RO}+\mathrm{RO}+\mathrm{O}_{2}$

21. $\mathrm{ROOH}=\mathrm{RO}+\mathrm{OH}$

22. RO decomposition

23. $\mathrm{QOOH}=$ cyclic ether $+\mathrm{OH}$ (cyclic ether formation)

24. $\mathrm{QOOH}=$ alkene $+\mathrm{HO}_{2}$ (radical site beta to $\mathrm{OOH}$ group)

25. $\mathrm{QOOH}=$ alkene + carbonyl $+\mathrm{OH}$ (radical site gamma to $\mathrm{OOH}$ group)

26. Addition of $\mathrm{O}_{2}$ to $\mathrm{QOOH}\left(\mathrm{QOOH}+\mathrm{O}_{2}=\mathrm{OOQOOH}\right)$

27. Isomerization of $\mathrm{OOQOOH}$ and formation of carbonylhydroperoxide and $\mathrm{OH}$

28. Decomposition of carbonylhydroperoxide to form oxygenated radical species and $\mathrm{OH}$

29. Cyclic ether reactions with $\mathrm{OH}$ and $\mathrm{HO}_{2}$

30. Decomposition of large carbonyl species and carbonyl radicals

For reaction class 11 , the addition of TPGME fuel radicals to $\mathrm{O}_{2}\left(\mathrm{R}+\mathrm{O}_{2} \leftrightarrow \mathrm{RO}_{2}\right)$, the rate constants were taken from the work of Sarathy et al. [22] on alkanes. The rate constants for fuel radical addition of $\mathrm{O}_{2}$ at the 3,7 and 11 sites (Fig. 6) were assumed to be the same as for a tertiary site in an alkane. Similarly, the rate constant for fuel radical addition to $\mathrm{O}_{2}$ at the 5, 9, 
and 13 sites were assumed to be the same as for a secondary site in an alkane. Site 1 was the only site where an "alkane" rule was not used. Here, the rate constant was assumed to be the same as for fuel radical addition to $\mathrm{O}_{2}$ for a primary site in DME $[8,9]$. Analogous reaction rate constant rules were used for reaction class 26 , hydroperoxy-alkyl radical addition to $\mathrm{O}_{2}\left(\mathrm{QOOH}+\mathrm{O}_{2} \leftrightarrow\right.$ OOQOOH) reactions. The next most important reaction class is 15 , the alkylperoxy radical $\left(\mathrm{RO} \dot{ }_{2}\right)$ isomerization reactions. For this reaction class, the activation energies were adjusted to account for the reduced $\mathrm{C}-\mathrm{H}$ bond energy when the relevant carbon was bonded to an $\mathrm{O}$-atom, Fig. 6. For this adjustment, it was assumed that the reduction in the bond strength compared to that in an $n$-alkane is reflected fully in a reduction in the activation energy of the reaction as given by Carstensen et al. [23]. Analogous reaction rate constant rules were used for reaction class 27 , the hydroperoxy-alkylperoxy $\left(\dot{\mathrm{O}}_{2} \mathrm{QOOH}\right)$ isomerization reactions. The concerted elimination reactions of $\mathrm{HO}_{2}$ radicals from $\mathrm{RO} \dot{ }_{2}$ radicals were included in the mechanism in reaction class 16 . For the rate constant assignments of the other reaction classes, they are given in the reaction mechanism (tpgme_mech_low-highT_vlq) included as Supplementary data, along with the sources of reaction rate constants for each class. The final mechanism consists of 553 species and 2720 reactions.

The chemical kinetic mechanism was analyzed using tools developed by McNenly [24] for reaction rates constants that exceeded theoretical limits in either the forward rate specified by the mechanism or the reverse rates computed using thermodynamic parameters. Any rates identified that exceeded these limits were analyzed and fixed by giving realistic rate estimates or by supplying improved thermodynamic property estimates.

As stated previously, isomer $3 \mathrm{a}$ was chosen as a surrogate to represent the 8 isomers present in the TPGME mixture (Fig. 1). It is interesting to qualitatively assess the low-temperature 
reactivity of the different isomers to obtain an indication of how the reactivity of isomer $3 \mathrm{a}$ compares to the reactivity of the other isomers. The reactivity of these isomers can be qualitatively compared by examining the reactivity of the various sites in the isomer and their relative position to each other. For example, it is known that 6-membered RO ${ }_{2}$ isomerizations (in reaction class 15) lead to low temperature chain branching by addition of $\mathrm{QOOH}$ to $\mathrm{O}_{2}$ (reaction class 26) and subsequent isomerization (reaction class 27) [25]. Thus the presence of two $\mathrm{CH}_{2}$ groups (secondary $\mathrm{C}-\mathrm{H}$ sites) that are $\beta$ to each other allows a fast 6 -membered ring $\mathrm{RO} \dot{ }_{2}$ isomerization that leads to low-temperature chain branching and indicates that such isomers would be particularly reactive. Isomers $1 \mathrm{a}$ and $1 \mathrm{~b}$ (Fig. 1) allow such RO ${ }_{2}$ isomerizations and are placed into the most reactive Group 1 (Fig. 1). Isomers $2 \mathrm{a}$ and 2c also allow such fast RÓ 2 isomerizations and $2 \mathrm{~b}$ has a fast $\mathrm{RO}_{2}$ isomerization involving a $\mathrm{CH}_{2}$ group and weak "ether-like" $\mathrm{C}-\mathrm{H}$ bonds on the terminal methyl group. However, these isomers also have features that are expected to lower their reactivity compared to Group 1 . The first feature in isomers $2 b$ and $2 c$ is a carbon next to an $\dot{O} \mathrm{H}$ group (the so-called $\alpha$ carbon in an alcohol [7]) that leads to unreactive products because abstraction of an $\mathrm{H}$-atom at this carbon will predominately lead to the production of an aldehyde and a $\mathrm{HO}_{2}$ radical rather than allow low-temperature chain branching to occur with production of reactive $\dot{O} \mathrm{H}$ radicals [7]. The second factor in isomer $2 \mathrm{a}$ and $2 \mathrm{c}$ is the presence of an $\mathrm{RO} \dot{ }_{2}$ isomerization between two tertiary sites (where methyl groups are attached). Six-membered ring $\mathrm{RO} \dot{ }_{2}$ isomerizations between two tertiary sites are the least reactive case because conventional low-temperature chain-branching to produce $\dot{\mathrm{O}} \mathrm{H}$ radicals does not occur because of the lack of a "weak $\mathrm{C}-\mathrm{H}$ bond" to abstract in the second isomerization (reaction class 27), and only alternative $\mathrm{RO} \dot{ }_{2}$ isomerization paths can occur that abstract alternative $\mathrm{H}$-atoms in the molecule. The third factor for isomer $2 \mathrm{~b}$ is the presence of a RO $\dot{\mathrm{C}}_{2}$ 
isomerization between a tertiary and a secondary site. This is because the tertiary site has no "weak $\mathrm{C}-\mathrm{H}$ bond" to abstract in the second isomerization (reaction class 27), this combination will give lower reactivity than an $\mathrm{RO}_{2}$ isomerization between two secondary sites. Because of these factors promoting and reducing reactivity, isomers $2 \mathrm{a}, 2 \mathrm{~b}$ and $2 \mathrm{~b}$ are put into reactivity

Group 2. In Group 3, isomer 3a has no fast 6-membered ring $\mathrm{RO}_{2}$ isomerizations between $\mathrm{CH}_{2}$ groups. It has slower 6-membered ring $\mathrm{RO}_{2}$ isomerizations between tertiary sites and $\mathrm{CH}_{2}$ groups and between a tertiary site and weak "ether-like" $\mathrm{C}-\mathrm{H}$ bonds. Isomer $3 \mathrm{a}$ also has a carbon next to an $\dot{\mathrm{O}} \mathrm{H}$ group that leads to relatively unreactive aldehyde and $\mathrm{HO}_{2}$ radical products. Thus, isomer 3a is expected to have a lower reactivity compared to Group 2 and is placed in Group 3. (Note that isomer $3 \mathrm{a}$ is the isomer selected as a surrogate in this study to represent all 8 isomers). Isomers $4 \mathrm{a}$ and $4 \mathrm{~b}$ have the least reactive 6 -membered ring $\mathrm{RO} \dot{ }_{2}$ isomerizations that occur between two tertiary sites. They also have a lower reactivity $\mathrm{RO}_{2}$ isomerization between tertiary and $\mathrm{CH}_{2}$ site. Additionally, they have a carbon next to an $\dot{\mathrm{O}} \mathrm{H}$ group that leads to relatively unreactive aldehyde and $\mathrm{HO}_{2}$ radical products. Based on these lower reactivity factors, isomer $4 \mathrm{a}$ and $4 \mathrm{~b}$ are placed in Group 4. In conclusion based on the above notional arguments, isomer 3a (which is selected as the surrogate) is expected to be less reactive than isomers $1 \mathrm{a}-2 \mathrm{c}$, but more reactive than $4 a$ and $4 b$.

\subsection{Thermochemical Data}

The thermodynamic parameters for the species are very important because they are used to determine reverse rate constants. The $\mathrm{C}_{1}-\mathrm{C}_{4}$ species thermodynamic parameters were taken from AramcoMech1.3 [15]. The THERM [26] software was used to compute the thermochemical properties of TPGME-related species. The THERM database contains about 500 group values that are needed to account for the many possible molecular structures considered by its users and 
are mainly derived from J.W. Bozzelli (private communication, 2001) and from Benson [27]. Burke et al. [28] recently optimized fifty-one group values using experimental and theoretical values of thermodynamic properties of $46 \mathrm{C}_{1}-\mathrm{C}_{4}$ alkanes, alkenes, alcohols, hydroperoxides and their related radicals. These new group values have been adapted in the present study to provide more accurate thermodynamic property estimates. In the case of the TPGME molecule, 4 of the 6 groups that comprise the molecule use the newly optimized group values. The updated THERM database was then used to compute the thermodynamic properties of the TPGME-related species. The thermodynamic parameters and a species dictionary are available as Supplementary data. The thermodynamic parameters were analyzed for discontinuities in their thermodynamic functions which cause kinetic solver issues using to a tool by McNenly et al. [29]. Discontinuities were found and were fixed, improving the computational efficiency when using the mechanism and thermodynamic parameter files in combustion codes.

\section{Results and Discussion}

\subsection{Experimental Data and Model Validation}

The current chemical kinetic model was used to predict ignition delay times for the six experimental conditions described in Table 1 spanning a range of temperatures (980-1545 K), pressures (10 and $20 \mathrm{~atm})$, and equivalence ratios (0.5, 1.0 and 2.0) keeping the molar percent of TPGME constant at $0.25 \%$. The dilute mixture composition was chosen for the experiments due to the low vapor pressure of TPGME. While the mixtures studied may not directly compare to conditions during use of TPGME in an engine, the data is novel and provides the model its first practical targets to test the proposed thermochemistry and kinetics.

Chemkin-Pro [30] was used for all modeling results presented. The shock tube results were simulated as a constant-volume, homogeneous batch reactor since the initial pressure profile in a 
shock tube can be approximated by a constant volume process up to the time of auto-ignition [31]. The ignition delay times extracted from the simulated pressure histories were defined in the same fashion as they were in the experiments (described in the Experimental section).

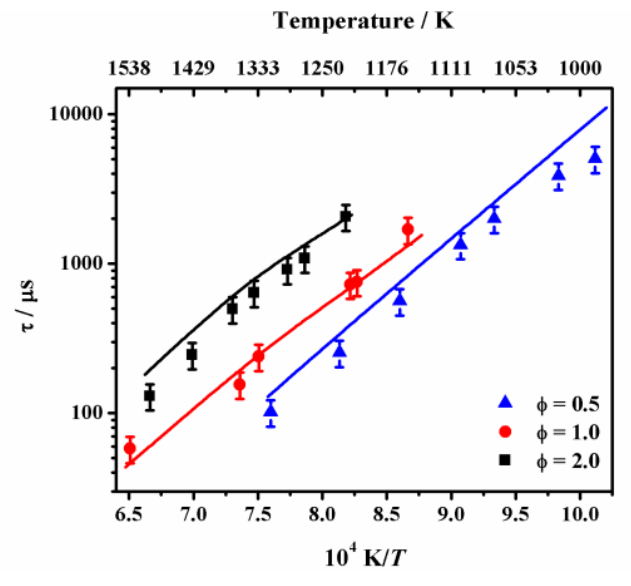

(a) $p_{5}=10 \mathrm{~atm}$

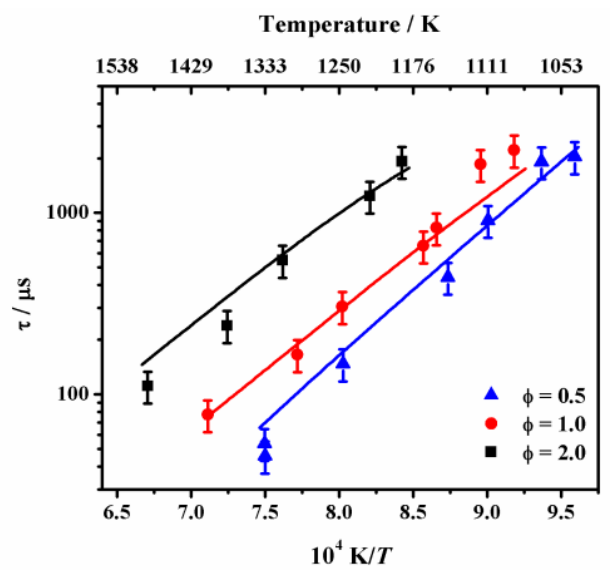

(b) $p_{5}=20 \mathrm{~atm}$

Figure 7: Ignition delay times measured in the shock tube (symbols) and simulated with the detailed chemical kinetic model presented here (lines) as a function of inverse temperature. The plots show the influence of changing equivalence ratio.

Figure 7 presents the ignition delay times measured at 10 and 20 atm showing the influence of equivalence ratio. At both pressures, ignition delay time decreases as equivalence ratio decreases ( $\left[\mathrm{O}_{2}\right]$ increases). The model captures this trend observed in the experiments. The model is also able to predict the ignition delay times well, with some discrepancies at low-temperatures for $\varphi=$ $0.5, p_{5}=10 \mathrm{~atm}$.

Brute force sensitivity analyses were performed for all three equivalence ratios at $T_{5}=1250 \mathrm{~K}$, and $p_{5}=10$ and 20 atm. The sensitivity analysis tool used was developed by McNenly et al. [29] For each of the six experimental conditions, the tool performed 4865 ignition calculations to compute the ignition delay time when both the forward and reverse rate was individually 
changed by a factor two. For each of the shock tube conditions, this took about 11 minutes on 48 Intel Xeon cores. In comparison, the calculation for each of the experimental conditions would take about one week using conventional software. A sensitivity coefficient was defined as:

$$
S=\frac{\ln \left(\tau_{+} / \tau_{-}\right)}{\ln \left(k_{+} / k_{-}\right)}=\frac{\ln \left(\tau_{+} / \tau_{-}\right)}{\ln (2 / 0.5)}
$$

$\tau_{+}$is the ignition delay after multiplying an individual A-factor by two and $\tau_{-}$is the ignition delay after dividing the same A-factor by a factor of two. A positive sensitivity coefficient indicates that increasing the reaction rate increases the ignition delay time (the reaction is inhibiting) and a negative sensitivity coefficient indicates that the reaction is accelerating.

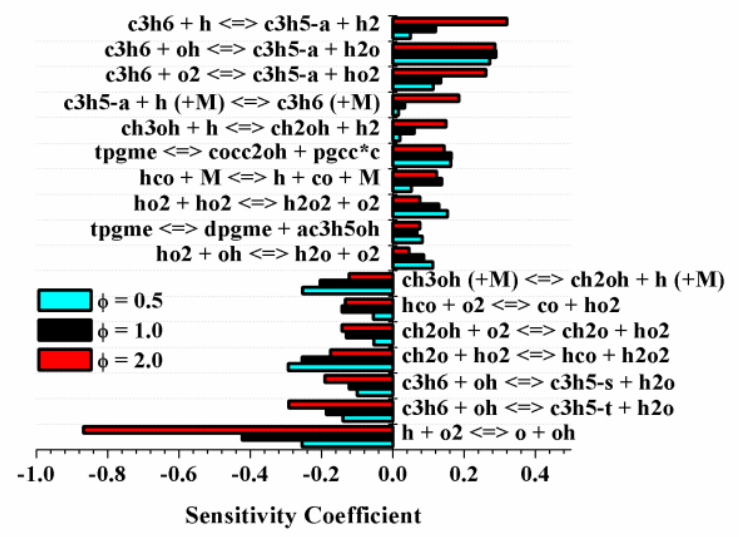

(a) $T_{5}=1250 \mathrm{~K}, p_{5}=10 \mathrm{~atm}$

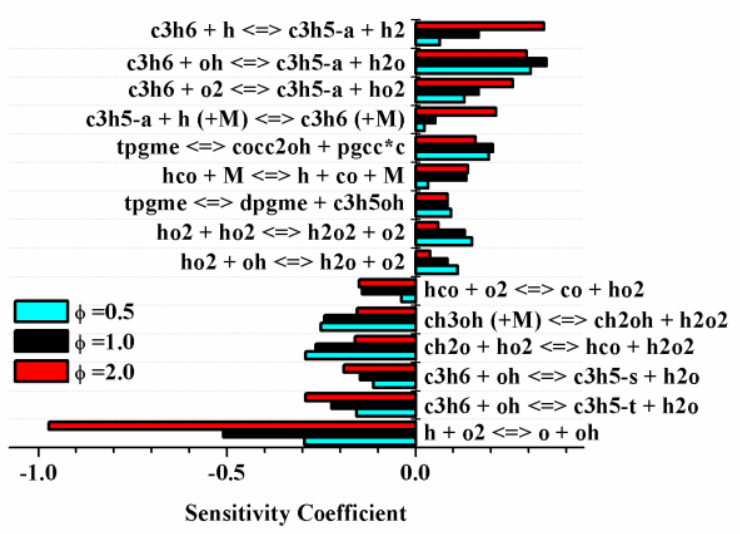

(b) $T_{5}=1250 \mathrm{~K}, p_{5}=20 \mathrm{~atm}$

Figure 8: Sensitivity analyses for 0.25\% TPGME, $\varphi=0.5,1.0$ and 2.0. (a) $10 \mathrm{~atm}$. (b) $20 \mathrm{~atm}$.

Only reactions with sensitivity coefficients with a magnitude above 0.01 are shown.

The sensitivity analyses results are shown in Fig. 8. At $\varphi=1.0$ and 2.0 and at both pressures the reaction $\dot{\mathrm{H}}+\mathrm{O}_{2} \leftrightarrow \ddot{\mathrm{O}}+\dot{\mathrm{O}} \mathrm{H}$ is the most sensitive one. While for $\varphi=0.5$ this reaction is still one of the most promoting reactions, but is less so than H-atom abstraction from formaldehyde by 
hydroperoxyl radicals. In general, reactions involving $\mathrm{HO}_{2}$ become more sensitive as the equivalence ratio is decreased. This is due to the effect on the main reaction that controls $\mathrm{HO}_{2}$ concentration: $\dot{\mathrm{H}}+\dot{\mathrm{O}}_{2}(+\mathrm{M}) \leftrightarrow \mathrm{HO}_{2}(+\mathrm{M})$. According to Table 1, the equivalence ratio is decreased in the reactant mixtures by keeping the fuel concentration fixed and increasing the oxygen concentration. This means that as the equivalence ratio decreases, the hydrogen atom concentration $[\dot{\mathrm{H}}]$ (which is derived mainly from the fuel) is nearly constant, while the $\mathrm{O}_{2}$ concentration increases. Thus $\mathrm{HO}_{2}$ concentration increases with decreasing equivalence ratio. This effect of the $\mathrm{HO}_{2}$ concentration increasing with decreased equivalence ratio is confirmed in Fig. 9a and $9 \mathrm{~b}$ where the simulated $\mathrm{HO}_{2}$ mole fraction histories are plotted as a function of equivalence ratio. Note that the maximum concentration of $\mathrm{HO}_{2}$ increases significantly with decreasing equivalence ratio.

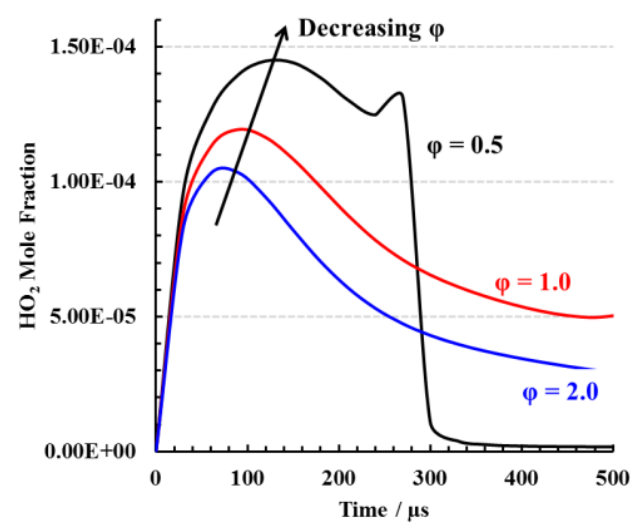

(a) $10 \mathrm{~atm}$

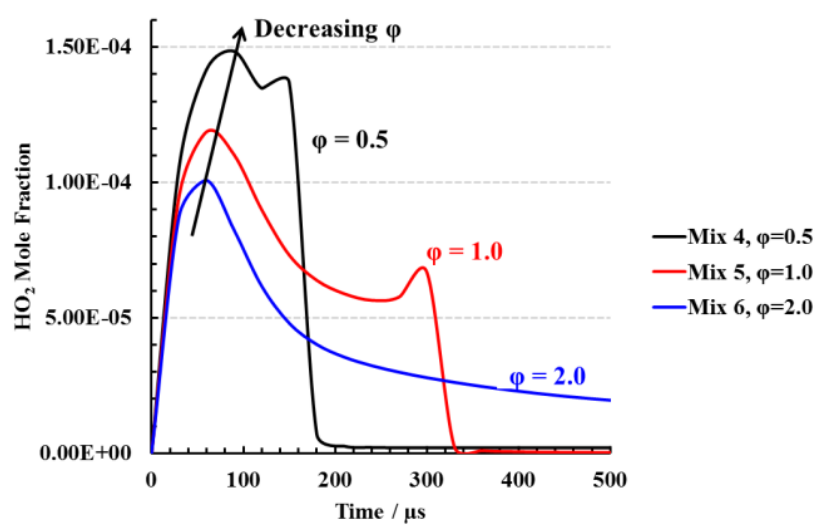

(b) $20 \mathrm{~atm}$

Fig. 9: Simulated $\mathrm{HO}_{2}$ mole fraction histories at $1250 \mathrm{~K}$ for reactant mixtures in Table 1. (a) 10 atm (b) $20 \mathrm{~atm}$

Six reactions shown to be sensitive have propene as a reactant or product, while the remaining ones are either reactions from the $\mathrm{H}_{2}$ sub-mechanism or reactions involving $\mathrm{C}_{1}$ species. Only two 
reactions that are found to be sensitive relate directly to TPGME species (see Fig. 13 which shows these reactions with their molecular structures):

$$
\begin{aligned}
& \text { TPGME } \leftrightarrow \text { COCC2OH }+ \text { PGCC }^{*} \mathrm{C} \\
& \text { TPGME } \leftrightarrow \text { DPGME }+\mathrm{aC}_{3} \mathrm{H}_{5} \mathrm{OH}
\end{aligned}
$$

These reactions are molecular eliminations and both are shown to be inhibiting, Fig 8 . All four of the products formed from reactions 1 and 2 decompose to produce propene. As discussed earlier in Section 3.1, these reactions have an uncertainty of 10 in their rate constant. Considering the sensitivity coefficients of about 0.2 in Fig. 8 for this reaction, this would give a factor of two uncertainty in ignition delay time. Measurements or $a b$ initio calculations of the reaction rate constants of these molecular elimination reactions are needed to reduce this uncertainty.

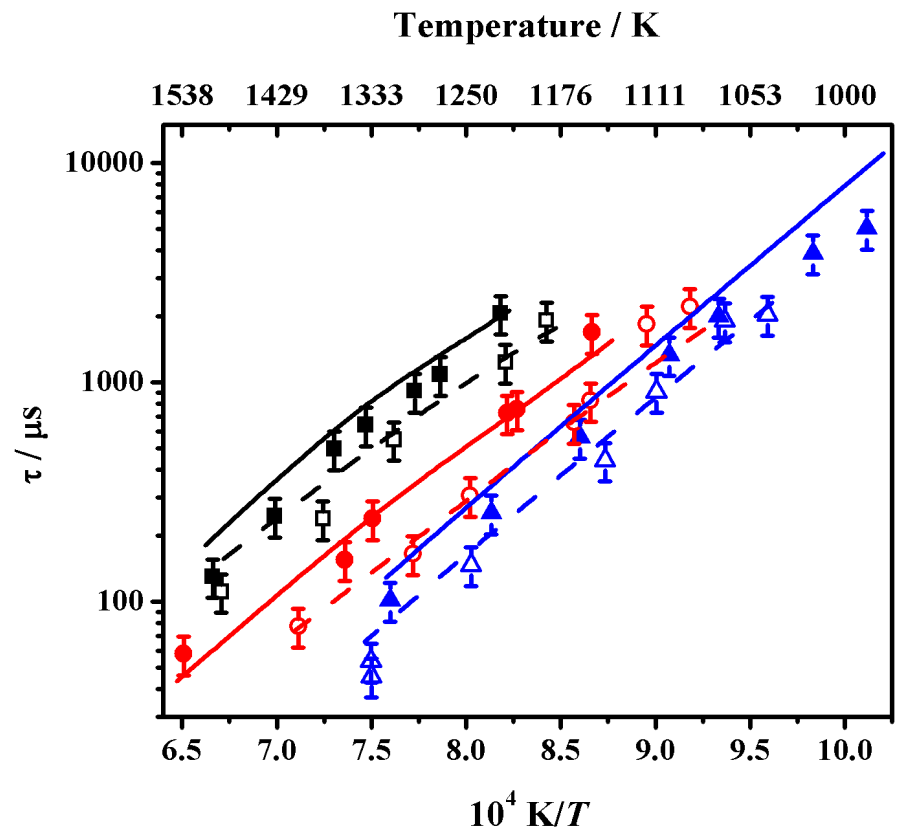

Figure 10: Influence of pressure and equivalence ratio. 0.25\% TPGME, Symbols are measurements and lines are model predictions. Filled symbols and solid lines are for $p_{5}=10$ 
atm, open symbols and dashed lines are $p_{5}=20 \mathrm{~atm}$. Squares $-\varphi=2.0$, circles $-\varphi=1.0$ and

$$
\text { triangles }-\varphi=0.5 \text {. }
$$

Figure 10 shows the influence of pressure and equivalence ratio of the ignition delay times. The experimental data shows excellent internal consistency for all equivalence ratios showing a decrease in ignition delay time with increasing pressure. Sensitivity analyses for $0.25 \%$ TPGME at $\varphi=1.0$ and $T_{5}=1250 \mathrm{~K}$ are compared as a function of pressure in Fig. 11.

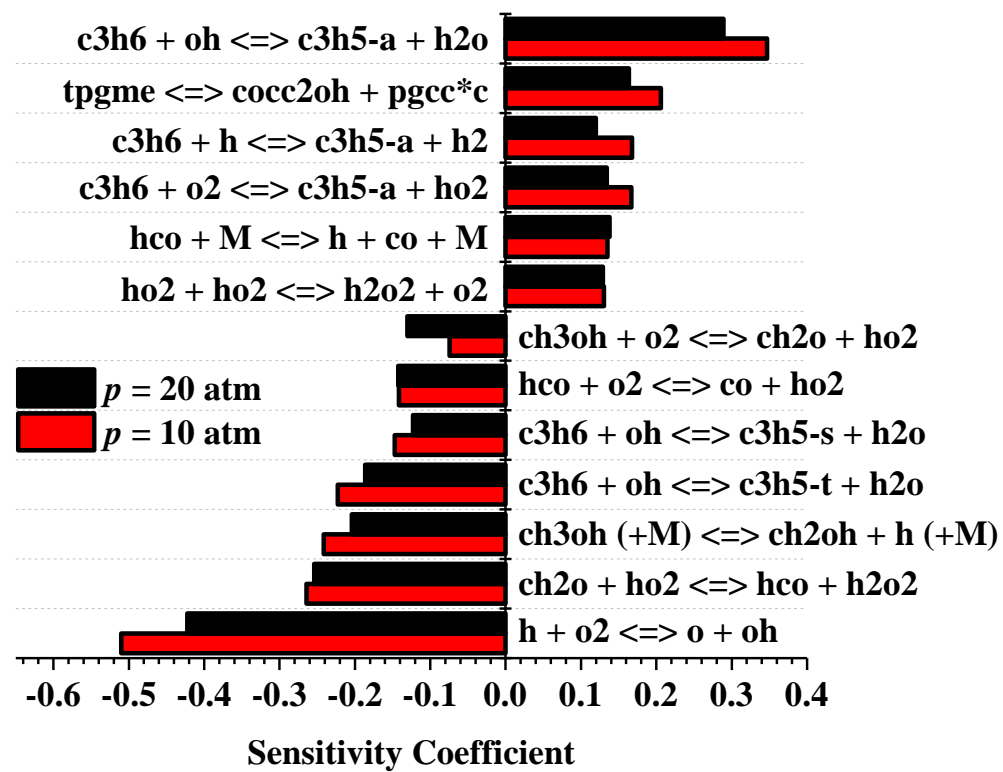

Figure 11: Sensitivity analyses for 0.25\% TPGME, $\varphi=1.0$ at $T_{5}=1250 \mathrm{~K}$ and pressures of 10 and 20 atm. Only reactions with sensitivity coefficients with a magnitude above 0.08 are shown.

There is no change in the reactions sensitive to predicting ignition delay times when the pressure is increased from 10 to $20 \mathrm{~atm}$. However, in the overall sensitivity analysis, reactions involving hydroperoxyl radicals are relatively more important at 20 atm, while at 10 atm reactions involving $\dot{\mathrm{H}}$ atoms are relatively more important. There is a high sensitivity to $\mathrm{H}$-atom abstraction reactions from propene. This leads to interest in the formation pathways of propene 
from TPGME. In order to more clearly illustrate the source of propene in the model a flux analysis was performed at the time of $20 \%$ TPGME consumption at $\varphi=1.0, p_{5}=19.4$ atm and $1167 \mathrm{~K}$. The major pathways forming propene at this time are outlined in Fig. 12.
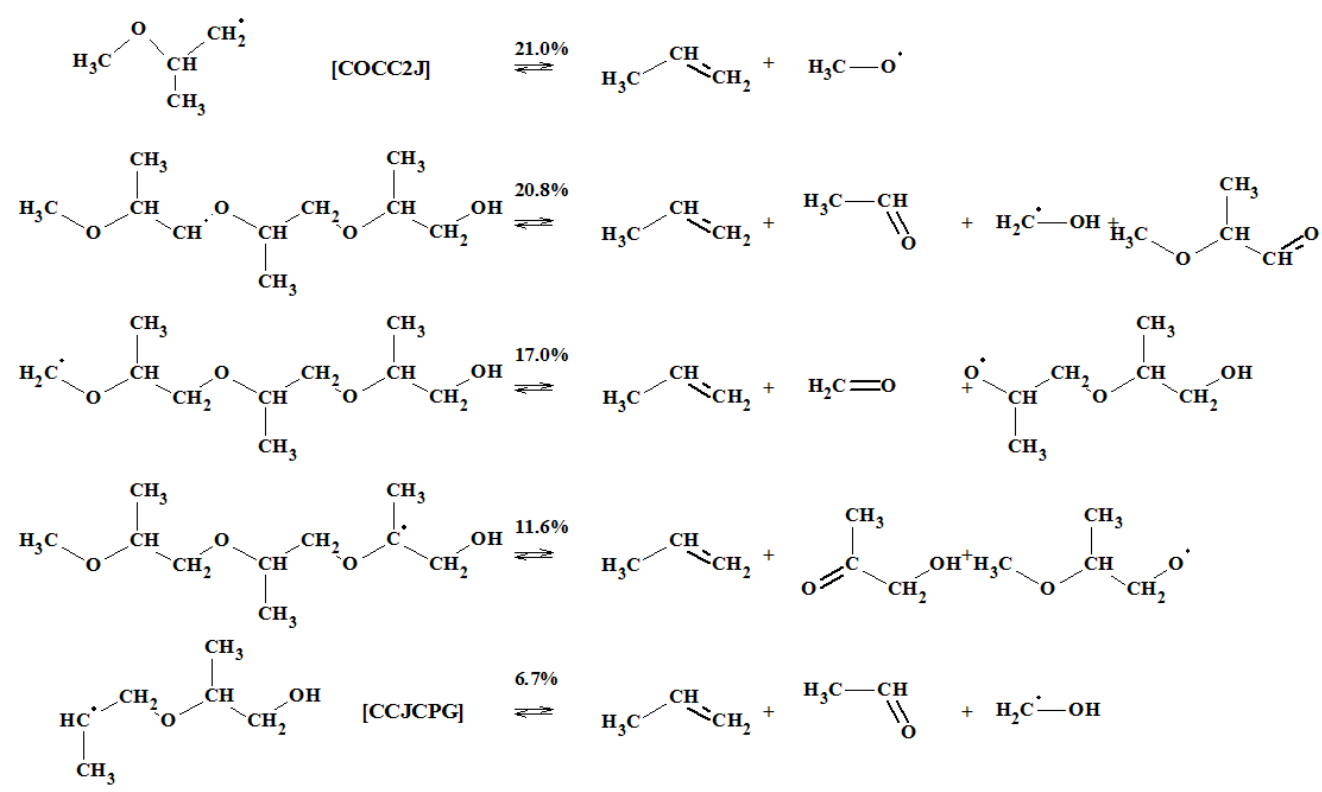

Figure 12: Propene production pathways at 20\% TPGME consumption under the conditions specified for mix no. 5 (Table 1). $\Phi=1.0, p=19.4 \mathrm{~atm}, T=1167 \mathrm{~K}$.

What is clear from Fig. 12 is that three of the major reactions forming propene are TPGME radical decomposition reactions. The other two propene formation reactions $(\beta$-scission of $\operatorname{cocc} 2 \mathrm{j}$ and decomposition of ccjcpg, refer to the species dictionary included in the Supplementary material for clarification of any structures discussed) are products of the decomposition of other TPGME radicals, namely tpgme-13 and tpgme-4, respectively (see Fig. 6 for radical naming scheme).

Regarding the consumption reactions of propene, which are shown to be sensitive to predicting the ignition delay times in Fig. 11, the current mechanism incorporates the calculation of Miller and Klippenstein [32] for $\mathrm{H}$-atom abstraction from propene by $\dot{H}$ atoms. For $\mathrm{H}$-atom abstraction 
from propene by hydroxyl radicals the measurement of Vasu et al. [33] is utilized for the total rate of $\mathrm{H}$-atom abstraction by hydroxyl radicals, and the branching ratio calculated by Zador $e t$ $a l$. [34] is incorporated to account for selectivity of abstraction at different sites in propene. The details of the propene sub-mechanism used in this model are discussed by Burke et al. [35]. The mechanism of oxidation of TPGME was explored using reaction path analysis, Fig. $13(\varphi=2.0$, $T_{5}=1187 \mathrm{~K}$ and $\left.p_{5}=18.4 \mathrm{~atm}\right)$. This analysis identifies that the main reactions consuming TPGME are molecular eliminations and $\mathrm{H}$-atom abstractions by $\dot{\mathrm{H}}$ atoms and $\dot{\mathrm{O}} \mathrm{H}$ and $\mathrm{HO}_{2}$ radicals. 


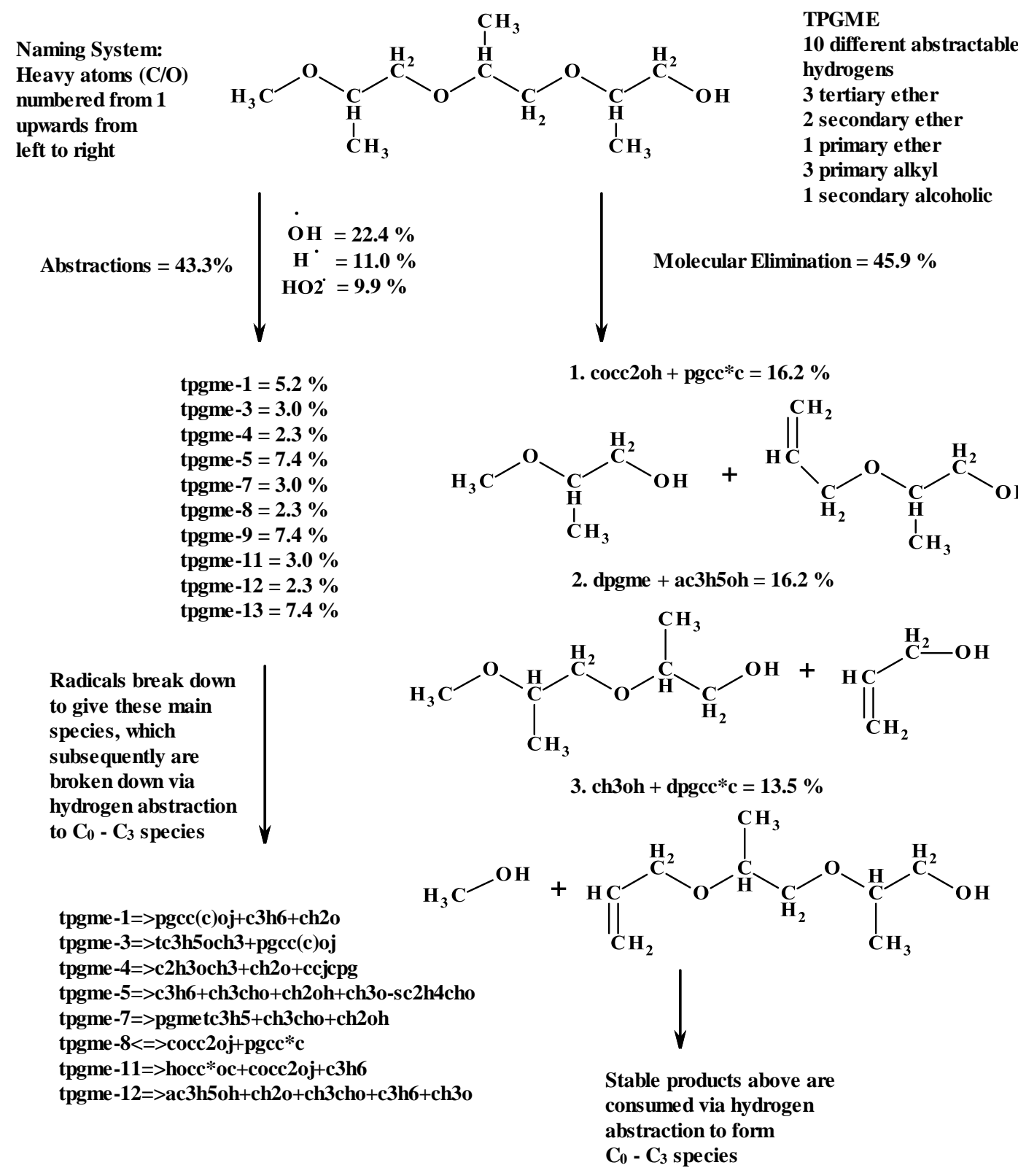

Full structures of radical species above is provided in the supplemental material

Figure 13: Reaction path analysis for $0.25 \%$ TPGME, $1.69 \% \mathrm{O}_{2}, 98.06 \%$ Ar for $p_{5}=18.41$ atm, $T_{5}=1187 \mathrm{~K}, \varphi=2.0$ and at $20 \%$ fuel consumed. Percentages represent net flux of reaction from the indicated species. The model presented herein (tpgme_mech_low-highT_vlq) is used to perform this flux analysis. 
The reaction path analysis (Fig. 13) shows that reactions 1 and 2 are the dominant molecular elimination reactions. $\mathrm{H}$-atom abstraction reactions by $\dot{\mathrm{O}} \mathrm{H}$ radicals are the most dominant abstraction reactions. As mentioned earlier there are 10 different abstraction sites (Fig. 6). The radical species most abundantly formed via $\mathrm{H}$-atom abstraction reactions are tpgme- $5,-9$ and 13, with $7.4 \%$ of the TPGME forming each of these radicals. These are formed by abstraction of a secondary $\mathrm{C}-\mathrm{H}$ bond adjacent to an oxygen atom (secondary ether). 5.2\% of the TPGME is consumed to form tpgme-1, where a primary ether $\mathrm{C}-\mathrm{H}$ bond is broken. While the tertiary ether C-H sites (tpgme-3, -7 and -11) account for 3.0\% of the consumption of TPGME each. The alkyl $\mathrm{C}-\mathrm{H}$ bonds of the methyl branches at sites tpgme- $4,-8$, and -12 consume $2.3 \%$ each. To understand this, it is important to consider the number of $\mathrm{H}$-atoms available for abstraction at each site. There are 6 secondary ether $\mathrm{H}$-atoms, 3 primary ether $\mathrm{H}$-atoms, 3 tertiary ether $\mathrm{H}$ atoms, and 9 primary alkyl $\mathrm{H}$-atoms. The bond strengths of these $\mathrm{C}-\mathrm{H}$ bonds are expected to follow this order:

$$
\text { tertiary ether }<\text { secondary ether }<\text { primary ether }<\text { primary alkyl }
$$

The reaction path analysis shows that more TPGME is consumed via the abstraction of secondary ether $\mathrm{H}$-atoms rather than tertiary ether $\mathrm{H}$-atoms. This is because there are twice as many secondary ether $\mathrm{H}$-atoms available to abstract.

The radicals of TPGME and the other species related to its combustion break down yielding the following main products: propene, formaldehyde, acetaldehyde, hydroxyl methylene, propenol, propenal, water, hydrogen, methane, hydrogen peroxide, and hydroxyl radicals.

\subsection{Low-Temperature Mechanism Analysis}

In order to analyze the low-temperature chemistry of TPGME postulated for the first time in this study, ignition delay time predictions were compared to the diesel surrogate $n$-heptane. Figure 14 
shows predicted ignition delay times for TPGME and $n$-heptane in "air" at 50 atm and $\varphi=0.5$, 1.0, and 2.0, using this model (tpgme_mech_low-highT_vlq) for TPGME, and Lawrence Livermore National Laboratory's (LLNL) most recent $n$-heptane model [36] to predict the $n$ heptane times.

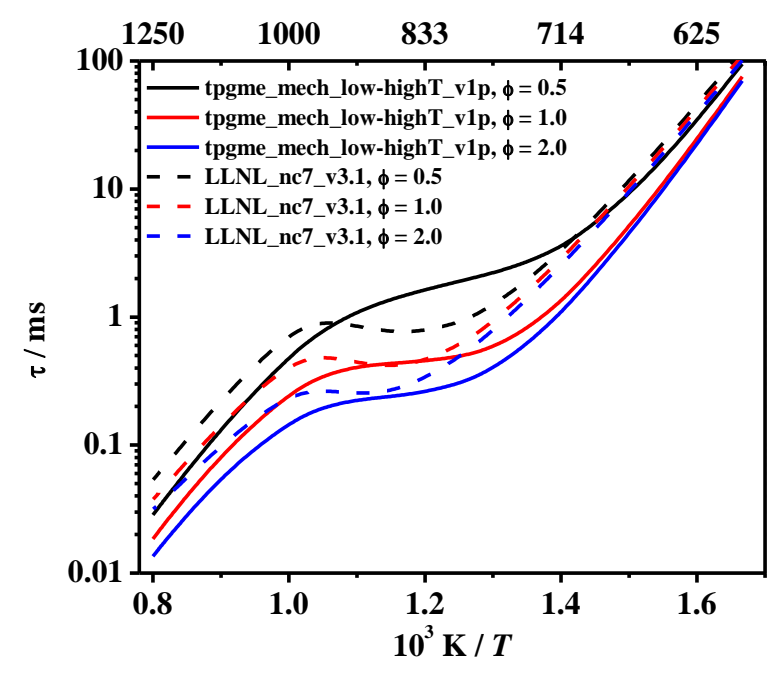

Figure 14: Comparison of ignition delay time predictions for 50 atm in "air" mixtures of TPGME and n-heptane. (tpgme_mech_low-highT_v1p used to predict TPGME times and LLNL_nc7_v3.1 used to predict n-heptane times.)

For $\varphi=1.0$ and 2.0 TPGME ignition delay times are faster than $n$-heptane over the entire temperature range $(600-1250 \mathrm{~K})$. In the temperature range $704-928 \mathrm{~K}$ at $\varphi=0.5$, ignition delay times are slightly slower for TPGME when compared to those predicted for $n$-heptane. These results are qualitatively consistent with the derived cetane numbers of $n$-heptane and TPGME (55 and 80, respectively [37]). A higher cetane number for TPGME (80) indicates it is more reactive than $n$-heptane (55) as is seen in Fig. 14, except for the $\varphi=0.5$ mixture. No data currently exists at low-temperatures to provide validation for the model. Hence this section is based purely on predictions of the current model. 


\subsection{Sensitivity analyses}

Brute force sensitivity analyses were performed for TPGME in "air" at $T_{5}=900 \mathrm{~K}, p_{5}=50$ atm and three equivalence ratios of $0.5,1.0$ and 2.0 , in order to illustrate the reactions controlling the prediction of first-stage and total ignition delay times. The results are shown in Fig. 15. The firstand second-stage ignition times were defined by the time when $50 \%$ of the temperature rise at their respective ignition occurs. Firstly, Fig. 15(a) shows the reactions that give high sensitivity to the first stage ignition. H-atom abstraction reactions by hydroxyl radicals are particularly important in predicting the first-stage ignition of TPGME. In addition, a number of the lowtemperature reactions proposed for the first time in this study are sensitive in predicting the firststage ignition of TPGME. These include reactions forming ketohydroperoxides and $\dot{O} \mathrm{H}$ radicals that are accelerating, and reactions that form cyclic ethers that are inhibiting. Secondly, Fig. 15(b) shows the reactions that give high sensitivity to the second stage ignition event. Only one of the reactions from the low-temperature oxidation mechanism for TPGME shows high sensitivity: TPGME—-9OO<=>TPGME-9OOH-5. H-atom abstraction reactions by hydroxyl radicals remain as two of the most promoting reactions. Both the most promoting and inhibiting reactions involve hydrogen peroxide, with the chain branching hydrogen peroxide decomposition being the most promoting and $\mathrm{H}$-atom abstraction by hydroxyl radicals form hydrogen peroxide forming water and hydroperoxyl radicals being the most inhibiting. As seen earlier in high temperature sensitivity results (Fig. 8 and 11), the sensitivity of reactions that involve HO ${ }_{2}$ increases with decreasing equivalence ratio. However, the molecular elimination reactions of TPGME, which showed significant sensitivity at high temperature, did not exhibit significant sensitivity at typical conditions for ignition in a diesel engine and thus do not appear on Fig. 15. 


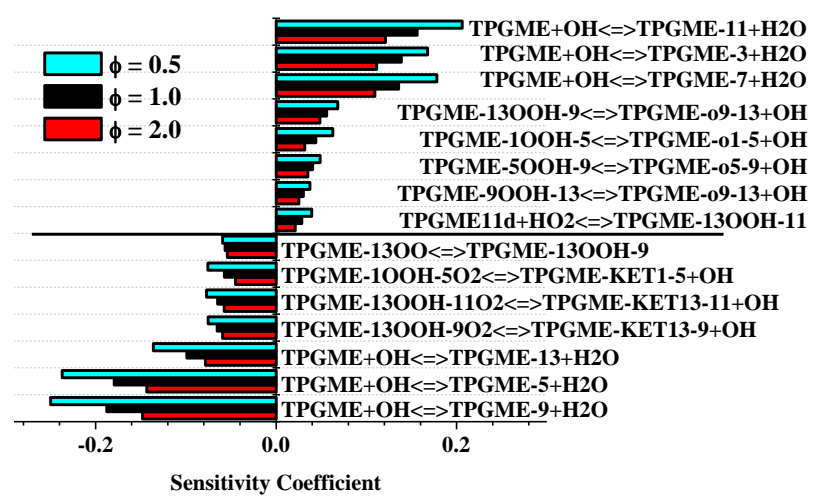

(a) Sensitivity to first stage ignition

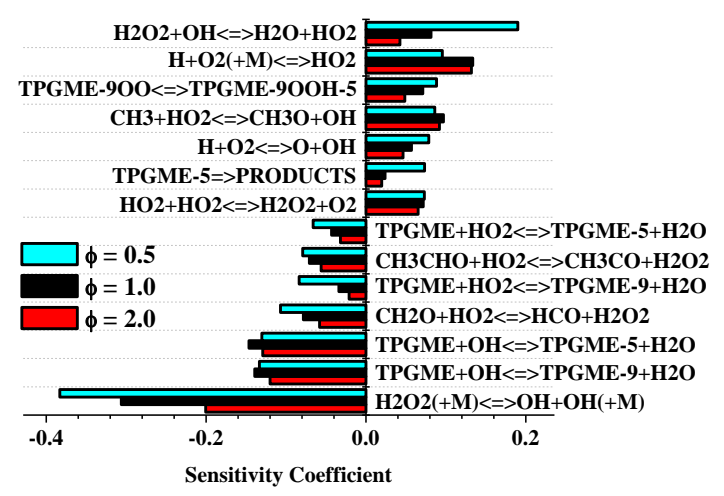

(b) Sensitivity to total ignition

Figure 15: Brute force sensitivity analyses for TPGME in "air" at $T_{5}=900 \mathrm{~K}$ and $p_{5}=50 \mathrm{~atm}$.

Only reactions with sensitivity coefficients with a magnitude above 0.02 are shown. The

reaction TPGME-5 $\Leftrightarrow$ PRODUCTS, refers to the reaction TPGME-

$5 \Leftrightarrow \mathrm{C} 3 \mathrm{H} 6+\mathrm{CH} 3 \mathrm{CHO}+\mathrm{CH} 2 \mathrm{OH}+\mathrm{CH} 3 \mathrm{O}-\mathrm{SC} 2 \mathrm{H} 4 \mathrm{CHO}$, in the model.

Considering TPGME's practical use as a soot reducing additive in diesel engines, it is particularly interesting to consider the difference in predictions of soot precursor species when compared with $n$-heptane which has often been used as a surrogate fuel for conventional diesel fuel e.g. [38]. Figure 16(a) presents the predicted mole fractions for some soot precursor species $\left(\mathrm{C}_{2} \mathrm{H}_{2}, \mathrm{C}_{2} \mathrm{H}_{4}\right.$, and $\left.\mathrm{C}_{3} \mathrm{H}_{6}\right)$ at $\varphi=3.0$ in "air", $p_{5}=50 \mathrm{~atm}$, and $T_{5}=770 \mathrm{~K}$ for both TPGME and $n$ heptane. These are well-known soot precursor species and are the same as used by Westbrook et al. [11] in their comparison of the soot precursor species formation of various oxygenated fuels to $n$-heptane under diesel engine conditions. The conditions of equivalence ratio, pressure and temperature are also the same as used by Westbrook et al. [11]. As shown in Fig. 16(a), there is less than half the quantity of acetylene predicted during TPGME oxidation when compared to $n$ heptane. There is more propene predicted for TPGME than for $n$-heptane, but there is a larger quantity of ethylene predicted for $n$-heptane than for TPGME. Figure 16(b) presents the total 
mole fraction of carbon in the precursor species predicted for $n$-heptane and TPGME. These numbers were determined by multiplying the mole fractions for acetylene, ethylene and propene by the number of carbon atoms in each molecule and summing the results to come to the total value of carbon mole fraction consumed via the formation of these soot precursor species. The results show that a significantly smaller quantity of carbon mole fraction is in the soot precursor species predicted for TPGME than for $n$-heptane.

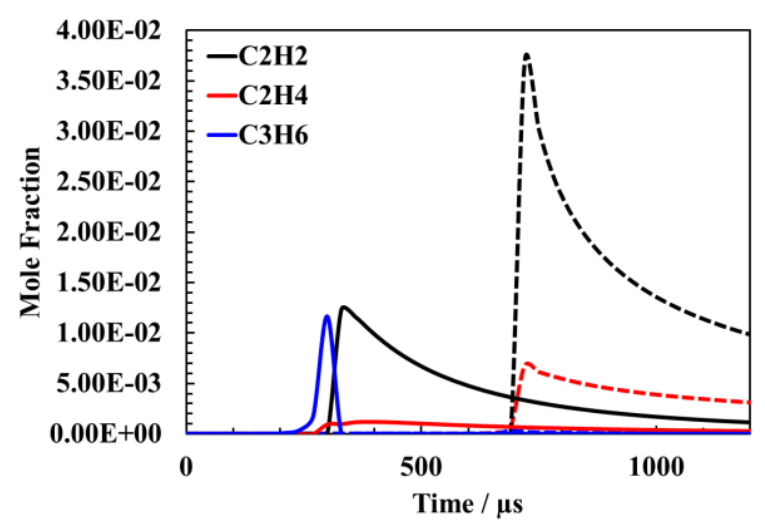

(a) Soot Precursors

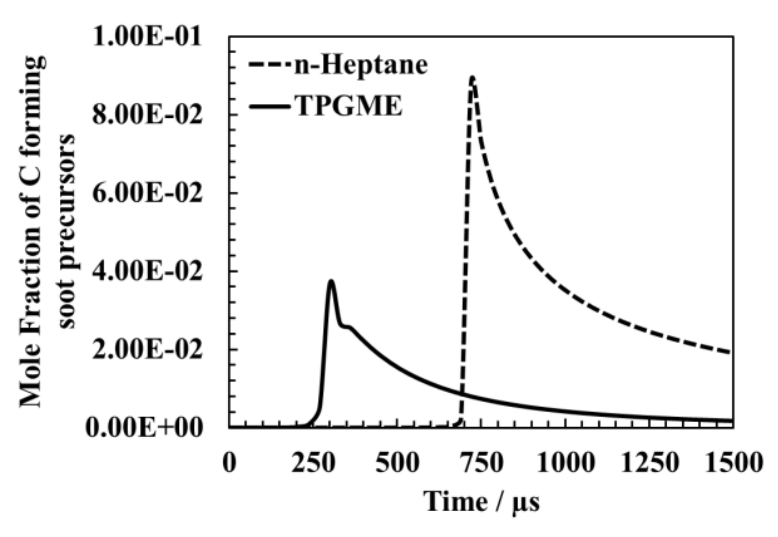

(b) Total mole fraction of $\mathrm{C}$ in soot precursors

Figure 16: Model predictions of soot precursor and total $C$ in the formed soot precursors. $\varphi=$ 3.0 in "air", $p_{5}=50 \mathrm{~atm}, T_{5}=770 \mathrm{~K}$. Solid lines represent TPGME predictions, dashed lines represent $n$-heptane predictions.

The soot reduction capability of TPGME is mainly due to the fact that when added as a mixture component to diesel, it increases the amount of oxygen present thereby making the fuel in "air" mixture leaner and reducing the amount of carbon available to form unsaturated hydrocarbon soot precursor species. As the mixture becomes more fuel-lean (closer to stoichiometric because diesel ignition occurs in fuel-rich mixtures), more $\dot{H}$ atoms and $\dot{O} \mathrm{H}$ radicals become available to consume any soot precursor species formed. The study of Westbrook et al. [11] concluded this in relation to TPGME also stating that the even distribution of oxygen atoms throughout the 
molecule ensures an efficient reduction in the amount of carbon available to form unsaturated hydrocarbon soot precursor species. No mechanism of scavenging of unsaturated hydrocarbons by TPGME and/or TPGME related species was found. These results offer insight as to why TPGME acts as an effective soot reducing additive when used during diesel engine combustion. 


\section{Conclusions and Future Work}

TPGME has previously been identified as an effective soot reducing additive. To date, no validated chemical kinetic model was available to predict the oxidation of this compound. Presented here is a surrogate chemical kinetic model for TPGME oxidation incorporating highand low-temperature reactions. The first fundamental experimental data (ignition delay times measured in a shock tube) for TPGME is presented, covering conditions of $0.25 \%$ TPGME, $\varphi=$ $0.5,1.0$, and 2.0, pressures of 10 and $20 \mathrm{~atm}$, and temperatures of $980-1545 \mathrm{~K}$. These data provide validation of the aforementioned chemical kinetic model. The model is capable of predicting these novel ignition delay times.

No experimental data currently exists to provide validation for the theoretical low-temperature reaction mechanism presented in this model. However a comparison of the ignition delay time predictions for TPGME and $n$-heptane are made for engine relevant conditions. The predictions of soot precursor species for TPGME and $n$-heptane are shown, compared, and evaluated for $\varphi=$ 3.0 in "air" mixtures at 50 atm and $770 \mathrm{~K}$. TPGME predicts a reduction in the quantity of acetylene by over a half when compared to $n$-heptane. In addition, when the total quantity of carbon mole fraction predicted in the soot precursor species is compared, it is clear that $n$ heptane predicts a larger quantity of carbon consumed to form soot precursor species than TPGME.

A surrogate model for the oxidation of TPGME at high- and low-temperatures is developed and is validated using the first ignition delay time measurements for this large oxygenated compound. Low-temperature data is required to further validate the model under lowtemperature conditions $(<980 \mathrm{~K})$, as the data presented showed negligible sensitivity to the lowtemperature pathways postulated; nonetheless the addition of these pathways was important in 
order to determine this (it should be noted that depending on the isomer distribution, the experimental results may differ as some isomers may have different reactivity). In order to further develop the kinetic mechanism for TPGME more experimental data are needed at low temperature in order to provide further constraints and test the chemical kinetics proposed in the current model. Additionally, quantum chemistry calculations of the reaction rates of key reactions noted in the sensitivity analysis, such as molecular elimination reactions of the fuel, $\mathrm{H}$ atom abstractions from the fuel by $\dot{\mathrm{O}} \mathrm{H}$ and $\mathrm{HO}_{2}$, and $\mathrm{RO}{ }_{2}$ isomerizations, would help improve the accuracy and predictivity of the model. The model developed represents a novel kinetic modeling study of a large oxygenated molecule containing a variety of chemical moieties including ether and alcoholic functional groups. It is also the only TPGME model which has been validated under any conditions to date.

\section{Acknowledgements}

The authors thank Dr. Eric Kurtz for helpful discussions and Dr. Jim Anderson for providing the treated fuel samples. The authors also thank Dr. Matt McNenly for help with the sensitivity calculations. The work at LLNL was supported by the U.S. Department of Energy, Vehicle Technologies Office (program manager Kevin Stork) and performed under the auspices of the US Department of Energy by Lawrence Livermore National Laboratory under Contract DEAC52-07NA27344. U. Burke and Prof. H. J. Curran would like to acknowledge funding from the

Irish Research Council. This material is based upon work supported by the Department of Energy under Award Number DE-EE0005386.

Disclaimer: This report was prepared as an account of work sponsored by an agency of the United States Government. Neither the United States Government nor any agency thereof, nor 
any of their employees, makes any warranty, express or implied, or assumes any legal liability or responsibility for the accuracy, completeness, or usefulness of any information, apparatus, product, or process disclosed, or represents that its use would not infringe privately owned rights. Reference herein to any specific commercial product, process, or service by trade name, trademark, manufacturer, or otherwise does not necessarily constitute or imply its endorsement, recommendation, or favoring by the United States Government or any agency thereof. The views and opinions of authors expressed herein do not necessarily state or reflect those of the United States Government or any agency thereof. 


\section{References}

1. C. J. Mueller, W. J. Pitz, L. M. Pickett, G. C. Martin, D. L. Siebers, C. K. Westbrook, SAE Technical Paper 2003-01-1791, 2003, doi:10.4271/2003-01-1791.

2. M. A. Gonzalez D, W. Piel, T. Asmus, W. Clark, J. Garbrak, E. Liney, M. Natarajan, D. W. Naegeli, D. Yost, E. A. Frame, J. P. Wallace, SAE Technical Paper 2001-01-3632, 2001, doi:10.4271/2001-01-3632.

3. J. Manin, S. Skeen, L. Pickett, E. Kurtz, J. E. Anderson, SAE Technical Paper 2014-012657, 2014. doi: 10.4271/2014-01-2657

4. (OECD) Propylene Glycol Ethers, SIDS Initial Assessment Report For SIAM 17; United Nations Environmental Programme (UNEP), <http://www.chem.unep.ch/irptc/sids/OECDSIDS/PGEs.pdf:> 2003.

5. $\quad$ S. Liang, Y. Zhou, H. Liu, T. Jiang, B. Han, Synth. Commun. 41 (2011) 891-897.

6. J. Li, Z. Zhao, A. Kazakov, M. Chaos, F. L. Dryer, J. J. Scire Jnr. Int. J. Chem. Kinet. 36 (2004) 566-575.

7. S. M. Sarathy, S. Vranckx, K. Yasunaga, M. Mehl, P. Oßwald, W. K. Metcalfe, C. K. Westbrook, W. J. Pitz, K. Kohse-Höinghaus, R. X. Fernandes, H. J. Curran, Combust. Flame, 159 (2012) 2028-2055.

8. S. L. Fischer, F. L. Dryer, H. J. Curran, Int. J. Chem. Kinet. 32 (2000) 713-740.

9. H. J. Curran, S. L. Fischer, F. L. Dryer, Int. J. Chem. Kinet. 32 (2000) 741-759.

10. K. Yasunaga, J. M. Simmie, H. J. Curran, T. Koike, O. Takahashi, Y. Kuraguchi, Y. Hidaka, Combust. Flame, 158 (2011) 1032-1036.

11. C. K. Westbrook, W. J. Pitz, H. J. Curran, J. Phys. Chem. A, 110 (2006) 6912-6922 doi:10.1021/jp056362g.

12. H. Nakamura, D. Darcy, M. Mehl, C. J. Tobin, W. K. Metcalfe, W. J. Pitz, C. K. Westbrook, H. J. Curran, Combust. Flame, 161 (2014) 49-64.

13. R. Mével, P. A. Boettcher, J. E. Shepherd, Chem. Phys. Lett. 531 (2012) 22-27.

14. C. Morley GASEQ, version 0.79, <http://www.arcl02.dsl.pipex.com/> (2013).

15. W. K. Metcalfe, S. M. Burke, S. S. Ahmed, H. J. Curran, Int. J. Chem. Kinet. 45 (2013) 638-675.

16. C-J Chen, J. W. Bozzelli, Eastern States Section Combust. Inst. (1999).

17. J. C. Brocard, F. Baronnet, H. E. O'Neal, Combust. Flame 52 (1983) 25-35.

18. C. M. Rosado-Reyes, W. Tsang, I. M. Alecu, S. S. Merchant, W. H. Green, J. Phys. Chem. A 117 (2013) 6724-6736.

19. R. Sivaramakrishnan, J. V. Michael, A. F. Wagner, R. Dawes, A. W. Jasper, L. B. Harding, Y. Georgievskii, S. J. Klippenstein, Combust. Flame, 158 (2011) 618-632.

20. R. S. Tranter, P. T. Lynch, C. J. Annesley, J. Phys. Chem. A. 116 (2012) 7287-7292.

21. J. Mendes, C.-W. Zhou, H. J. Curran, J. Phys. Chem. A, 118 (2014) 1300-1308.

22. S. M. Sarathy, C. K. Westbrook, M. Mehl, W. J. Pitz, C. Togbe, P. Dagaut, H. Wang, M. A. Oehlschlaeger, U. Niemann, K. Seshadri, P. S. Veloo, C. Ji, F. N. Egolfopoulos, T. Lu, Combust. Flame, 158 (2011) 2338-2357.

23. H.-H. Carstensen, A. M. Dean, O. Deutschmann, Proc. Combust. Inst. 31 (2007) 149-

157.

24. McNenly, M., Mechanism Tools, Personal communication, 2014.

25. C. K. Westbrook, Proc. Combust. Inst. 28 (2000) 1563-1577.

26. E. R. Ritter, J. W. Bozzelli, Int. J. Chem. Kinet. 23 (1991) 767-778.

27. S. W. Benson, Thermochemical Kinetics 2nd Ed. Wiley: New York, 1976. 
28. S. M. Burke, Development of a Chemical Kinetic Mechanism for Small Hydrocarbons.

$\mathrm{Ph}$. D. Thesis, National University of Ireland, Galway, Galway, 2014.

29. M. J. McNenly, R. A. Whitesides, D. L. Flowers, in: 8th US National Combustion

Meeting, University of Utah, Park City, UT, United States, 2013.

30. CHEMKIN-PRO 15112, Reaction Design: San Diego, 2011.

31. D. F. Davidson, R. K. Hanson, Int. J. Chem. Kinet. 36 (2004) 510-523.

32. J. A. Miller, S. J. Klippenstein, J. Phys. Chem. A, 117 (2013) 2718-2727.

33. S. S. Vasu, Z. Hong, D. F. Davidson, R. K. Hanson, D. M. Golden, J. Phys. Chem. A, 114 (2010) 11529-11537.

34. J. Zador, A. W. Jasper, J. A. Miller, Phys. Chem. Chem. Phys. 11 (2009) 11040-11053.

35. S. M. Burke, W. K. Metcalfe, O. Herbinet, F. Battin-Leclerc, F. M. Haas, J. Santner, F. L. Dryer, H. J. Curran, Combust. Flame, 161 (2014) 2765-2784.

36. M. Mehl, W. J. Pitz, C. K. Westbrook, H. J. Curran, Proc. Combust. Inst. 33 (2011) 193200.

37. J. Yanowitz, M. A. Ratcliff, R. L. McCormick, J. D. Taylor, M. J. Murphy, Compendium of Experimental Cetane Numbers, Report \# NREL/TP-5400-61693, National Renewable Energy Laboratory, (2014).

38. P. F. Flynn, R. P. Durrett, G. L. Hunter, A. O. Z. Loye, O. C. Akinyemi, J. E. Dec, C. K. Westbrook, Soc. Automotive Engineers Trans. 108 (3) (1999) 587-600. 


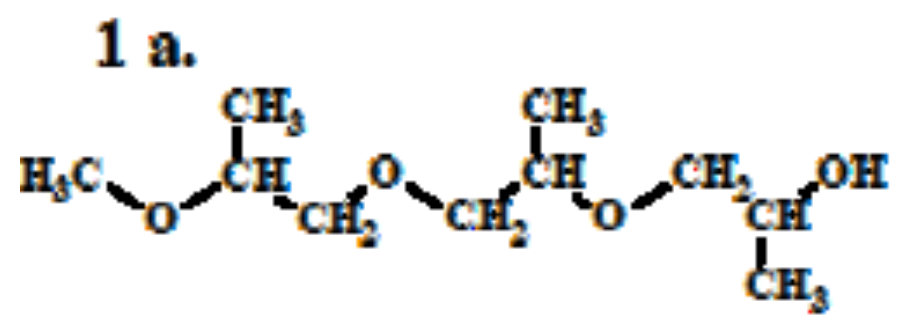

2 a.

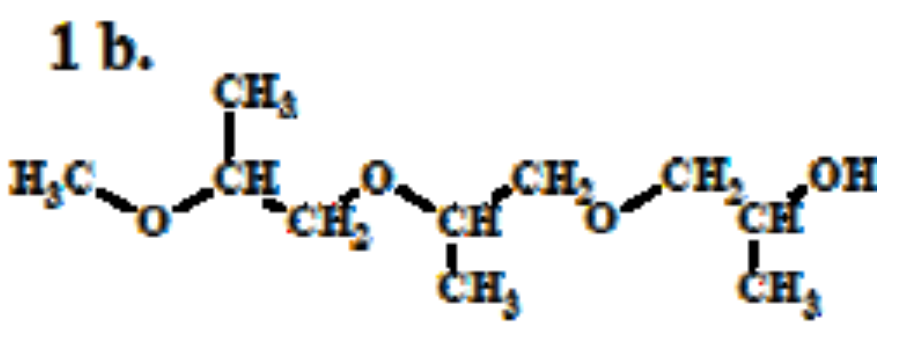

2 b.

$2 \mathrm{c}$.<smiles>COC(C)COCC(C)OC(C)CO</smiles><smiles>COC(C)COC(C)COC(C)CO</smiles>

4 a.<smiles>COC[C@@H](C)O[C@@H](C)C[C@H](C)O[C@H](C)CO</smiles>

$4 \mathrm{~b}$.<smiles>COC[C@@H](C)OC[C@@H](C)OC(C)CO</smiles> 


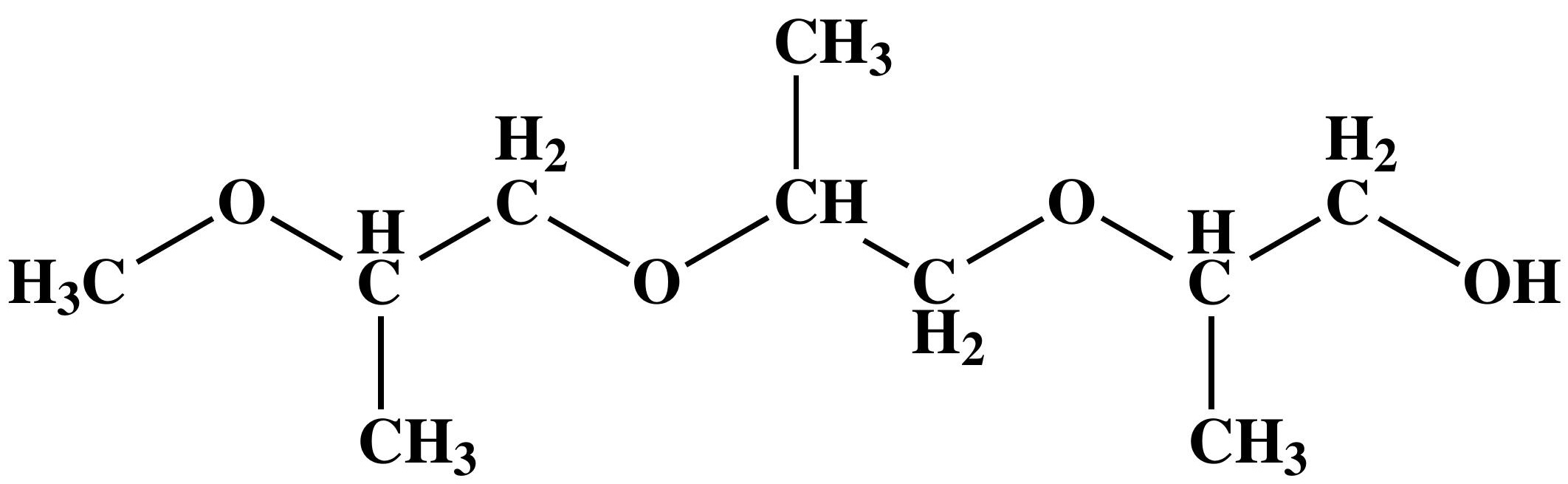

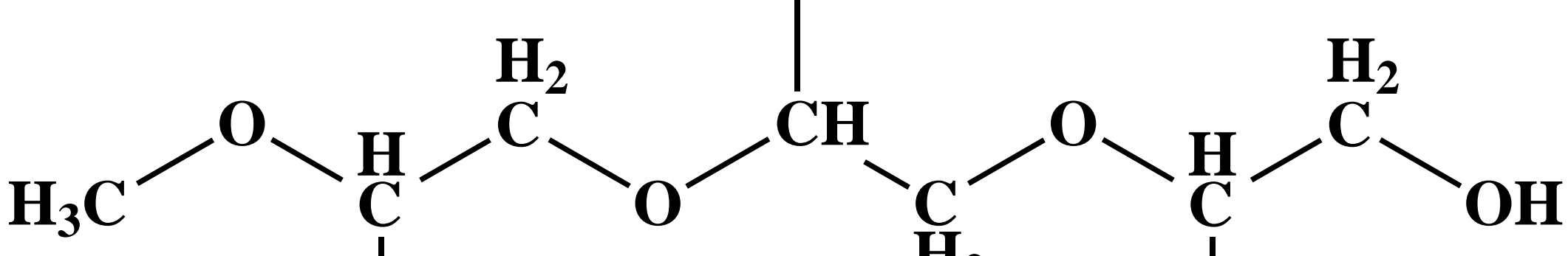
$\mathrm{CH}_{3}$

$\mathrm{CH}_{3}$ 


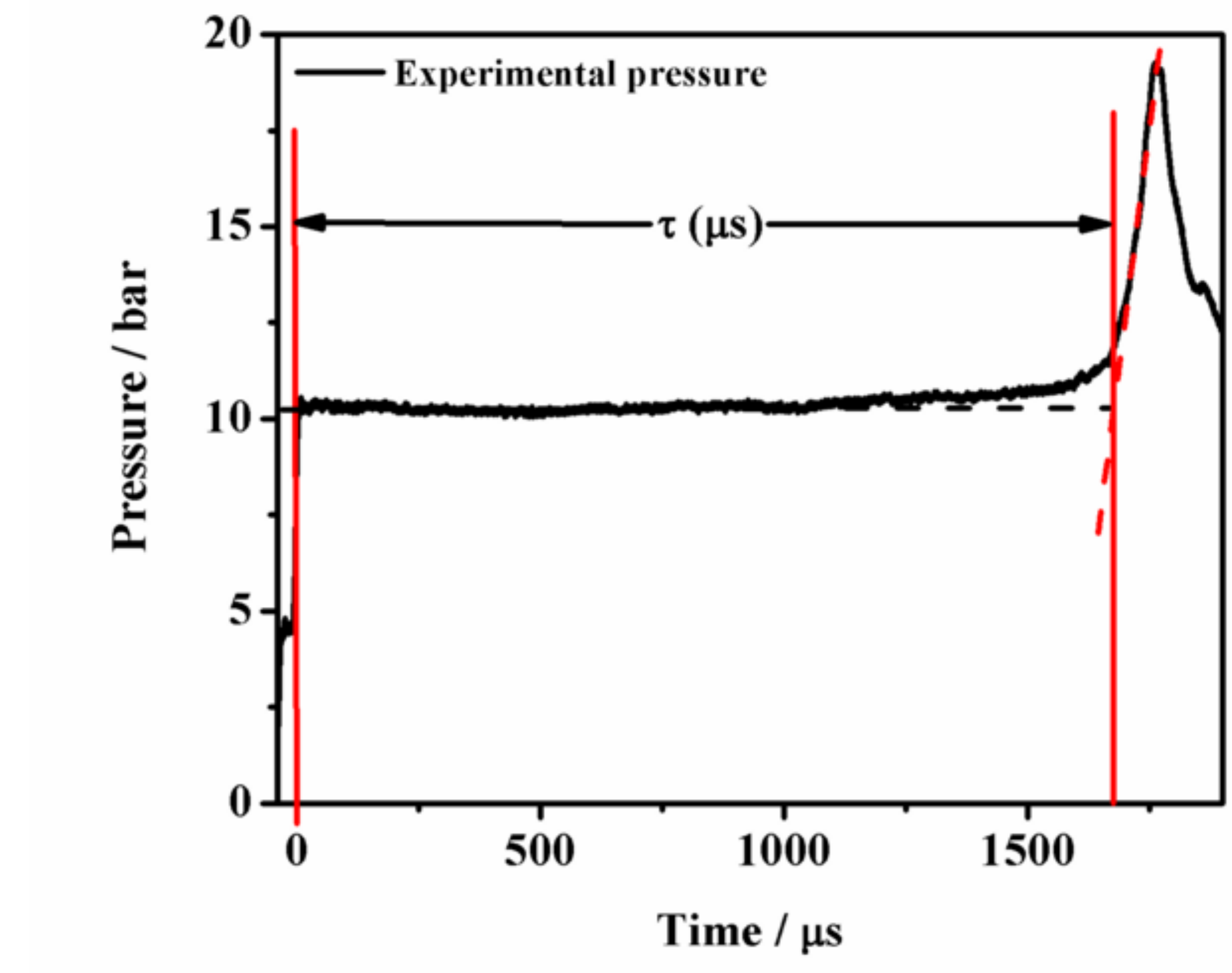

Time / $\mu$ s

.

.

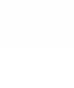

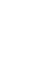




\section{Temperature / K}

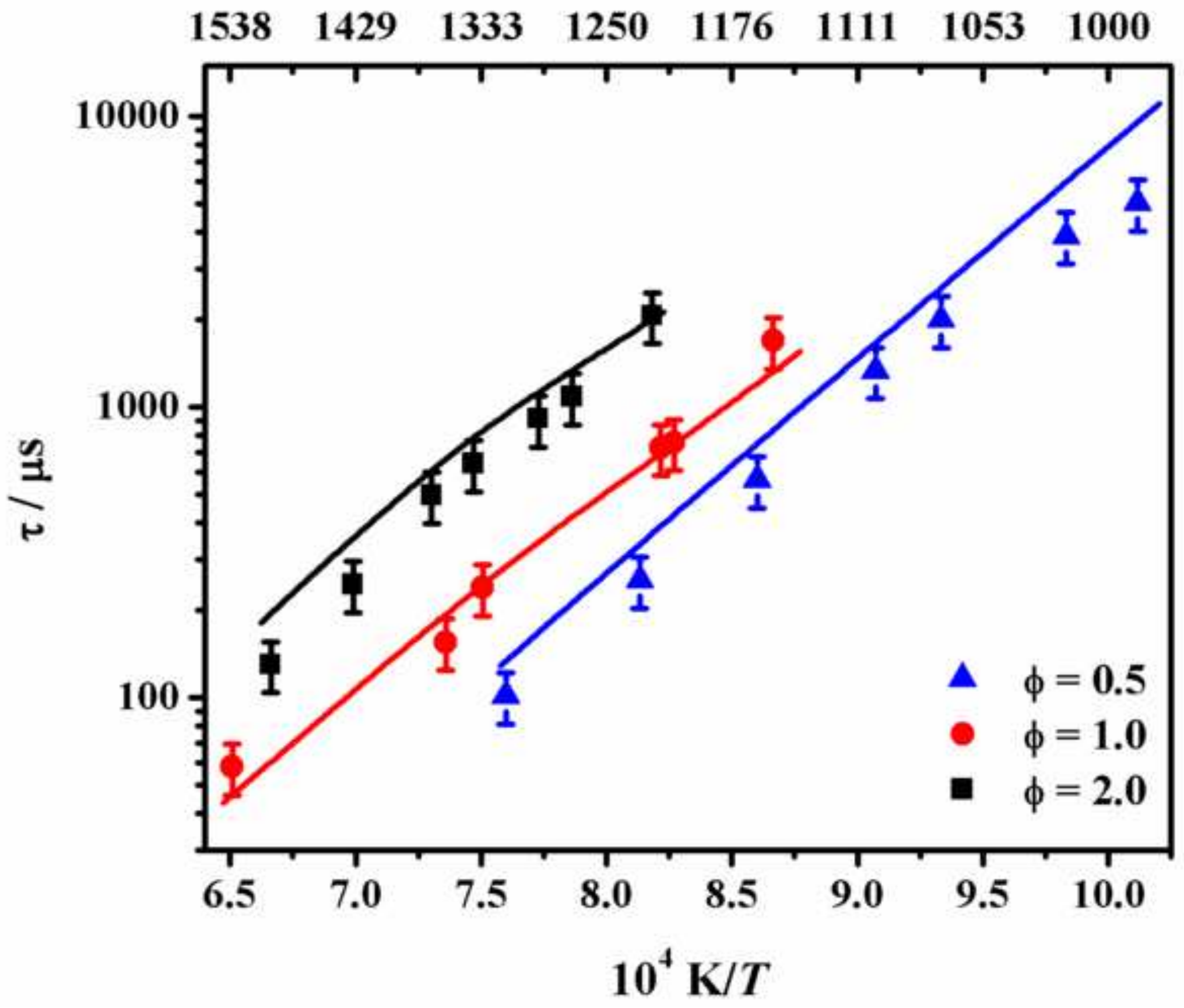


Temperature / K

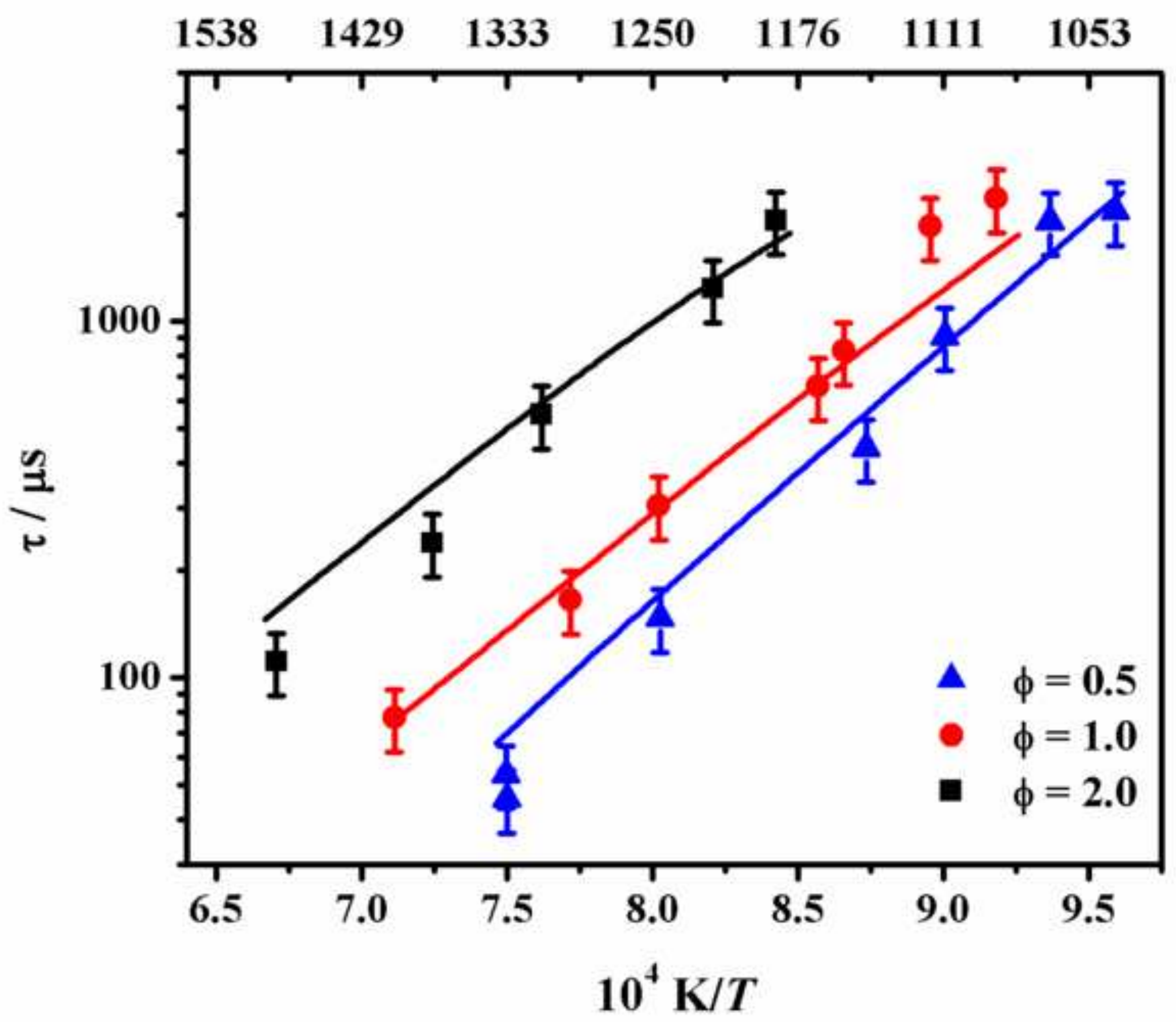




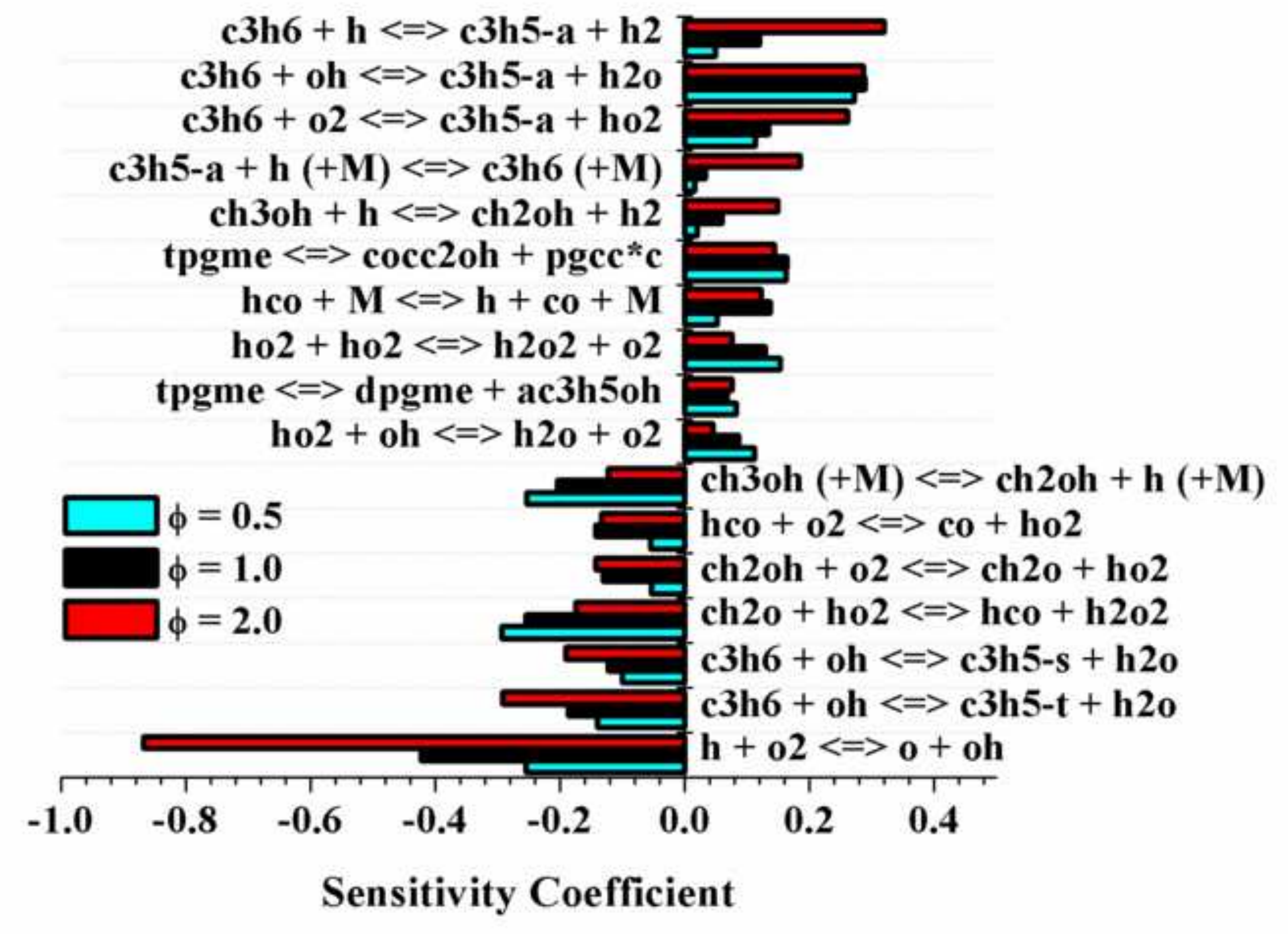

c3h6 + oh $\Leftrightarrow$ c3h5-a + h2o

c3h6 $+02<=>$ c3h5-a + ho2

$\operatorname{ch} 30 h+h \Leftrightarrow \operatorname{ch} 20 h+h 2$

hco $+\mathbf{M} \Leftrightarrow=\mathbf{h}+\mathbf{c o}+\mathbf{M}$

ho2 + ho2 $\Leftrightarrow$ h202 + o2

ho2 + oh $<=>$ h2o + o2

$\operatorname{ch} 20 h+02 \Leftrightarrow>\operatorname{ch} 20+$ ho2

ch2o + ho2 $\Leftrightarrow$ hco + h2o2

c3h6 + oh $\Leftrightarrow$ c3h5-s + h2o

c3h6 + oh $\Leftrightarrow$ c3h5-t + h2o

h + o2 $\Leftrightarrow>0+$ oh

$\begin{array}{rrrrrr}-1.0 & -0.8 & -0.6 & -0.4 & -0.2 & 0.0 \\ & & & \text { Sensitivity } & \text { Coefficient }\end{array}$ 


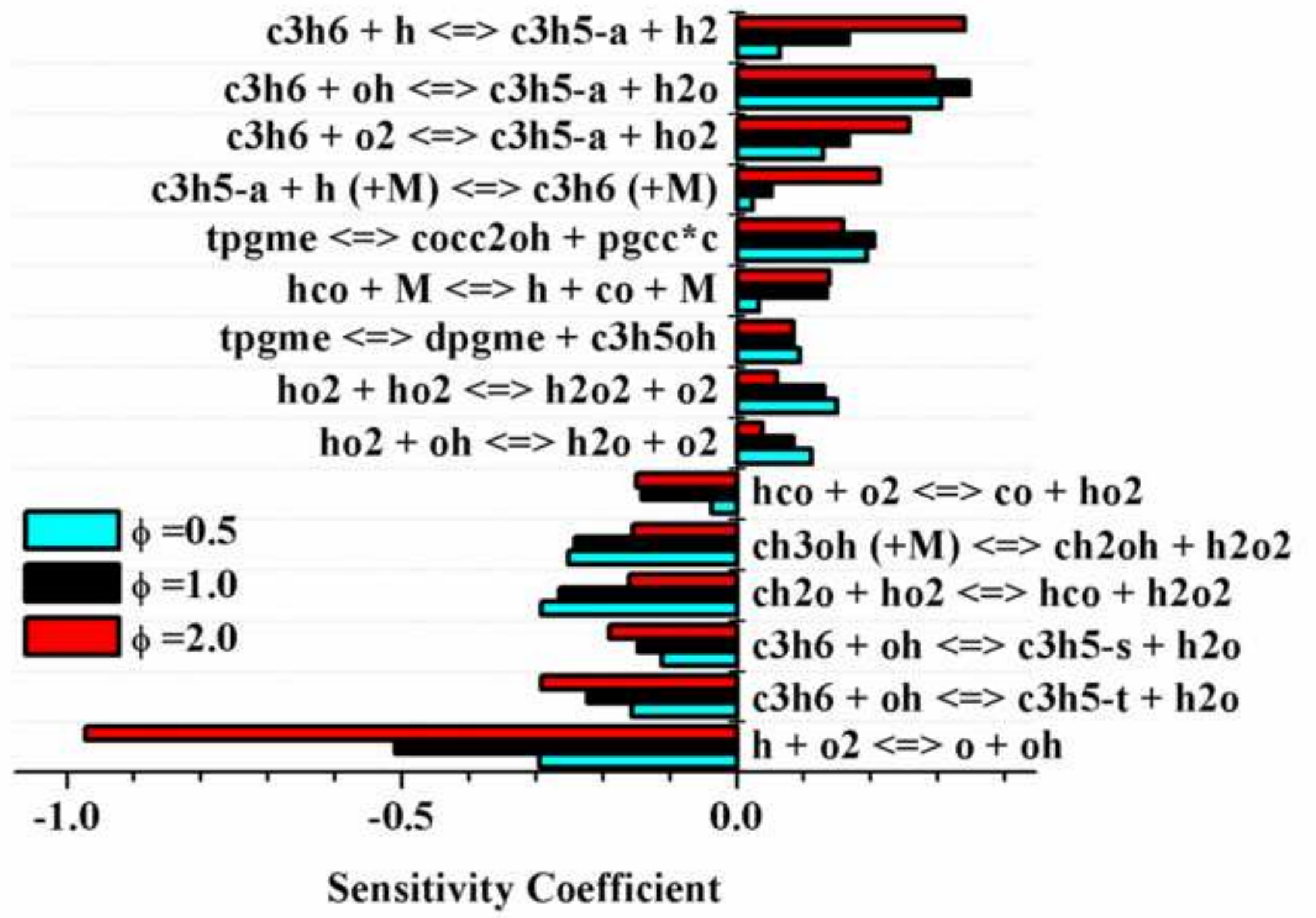
$\operatorname{ch} 30 h(+M)<\operatorname{ch} 20 h+h 202$ ch $20+$ ho2 $<=>$ hco + h2o2 c3h6 + oh $\Leftrightarrow$ c3h5-s + h2o c3h6 + oh $\Leftrightarrow$ c 3h5-t + h2o $\mathrm{h}+\mathrm{o2}<=>0+$ oh

Sensitivity Coefficient 


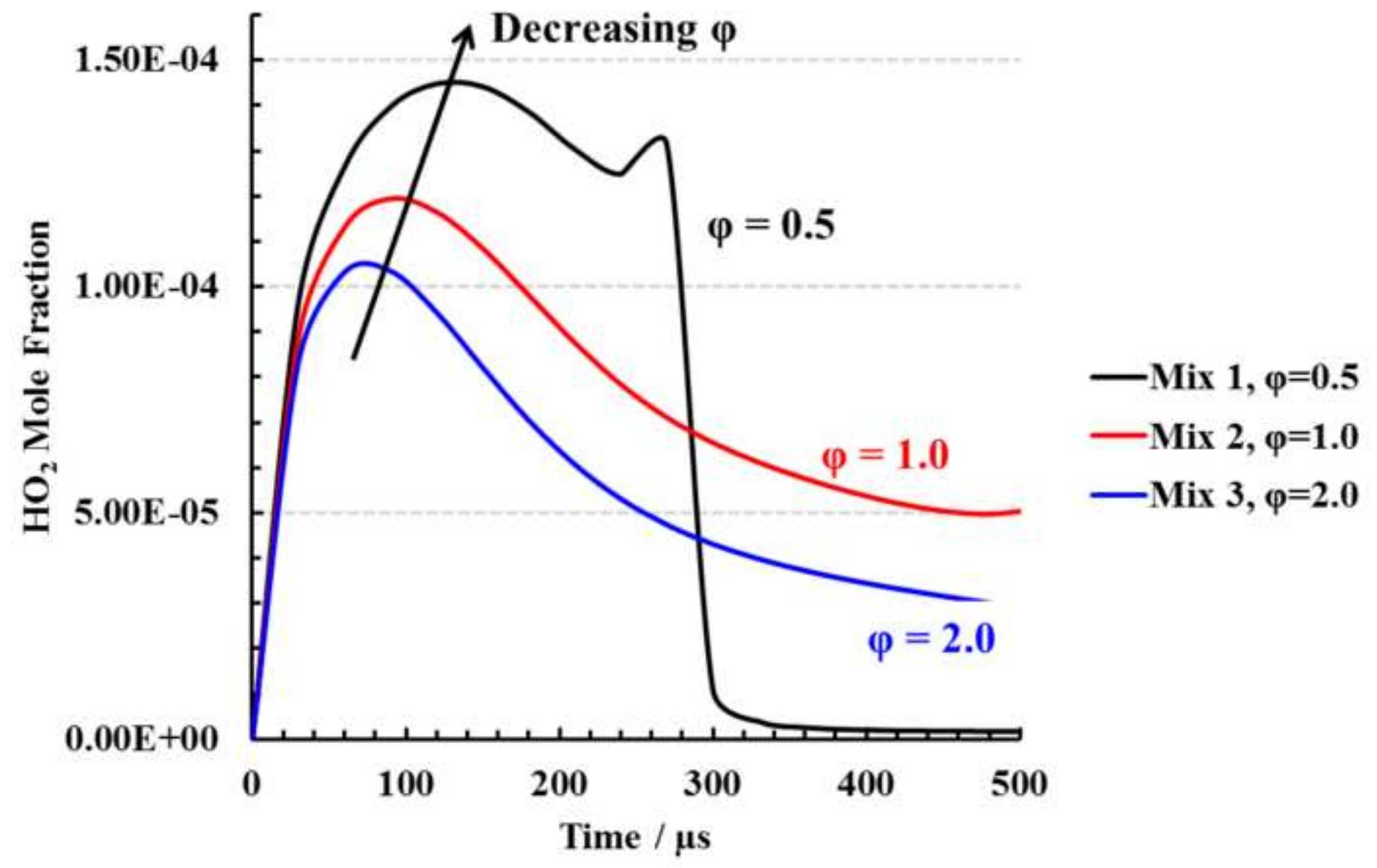




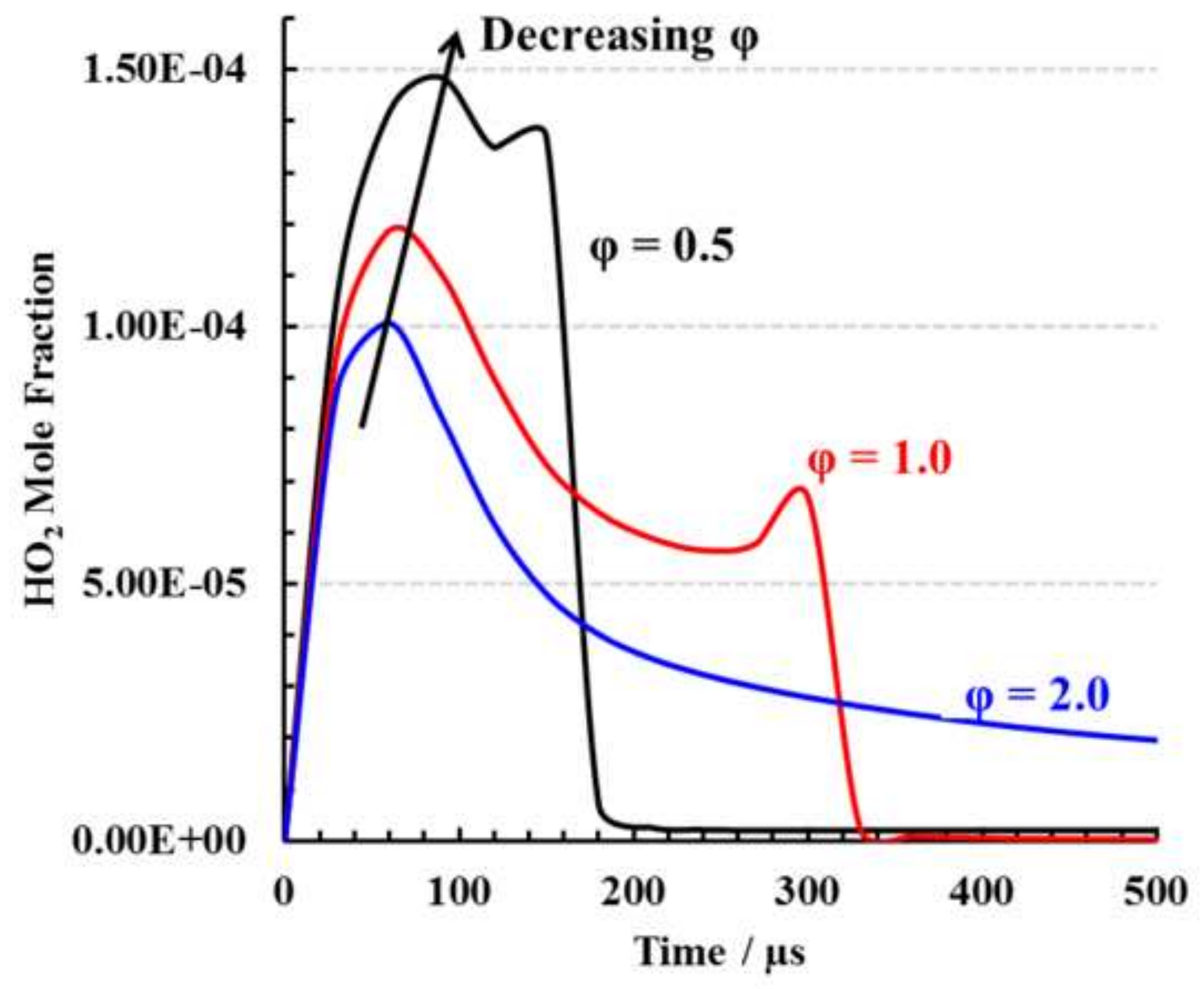

$-\operatorname{Mix} 4, \varphi=0.5$

$-\operatorname{Mix} 5, \varphi=1.0$

$\longrightarrow$ Mix $6, \varphi=2.0$

$0.00 \mathrm{E}+00$

Time $/ \mu \mathrm{s}$ 


\section{Temperature / K}

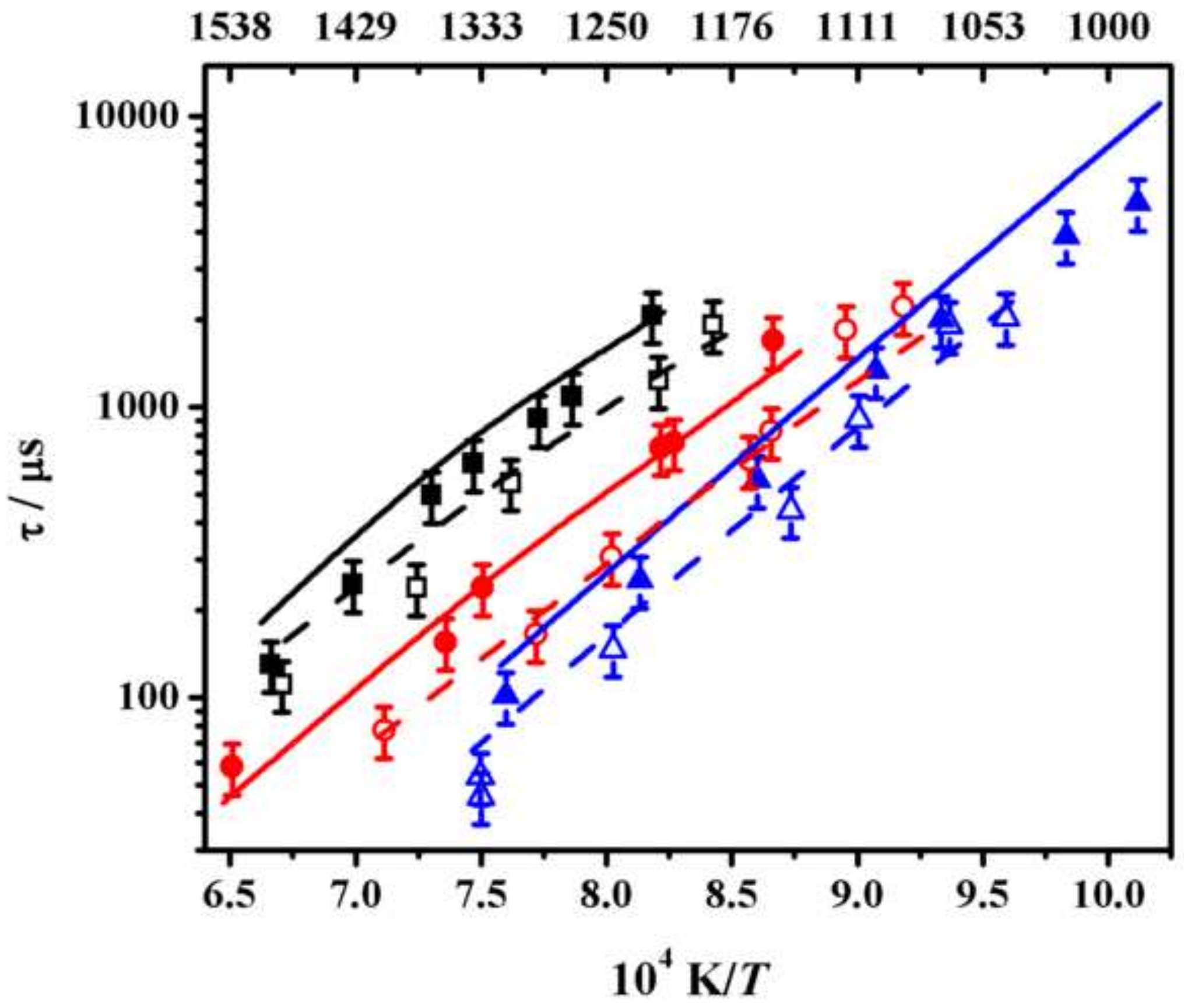


$\mathrm{H}_{3} \mathrm{C}^{\circ}{ }_{\mathrm{CH}_{3}}^{\mathrm{Ch}^{\mathrm{CH}}}$

[COCC2J]

$21.0 \%$

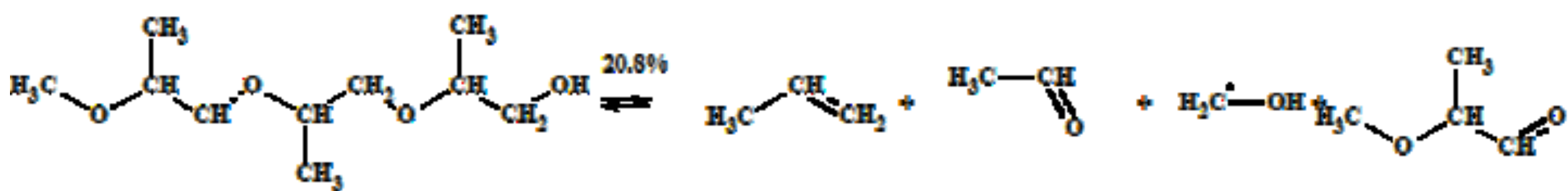

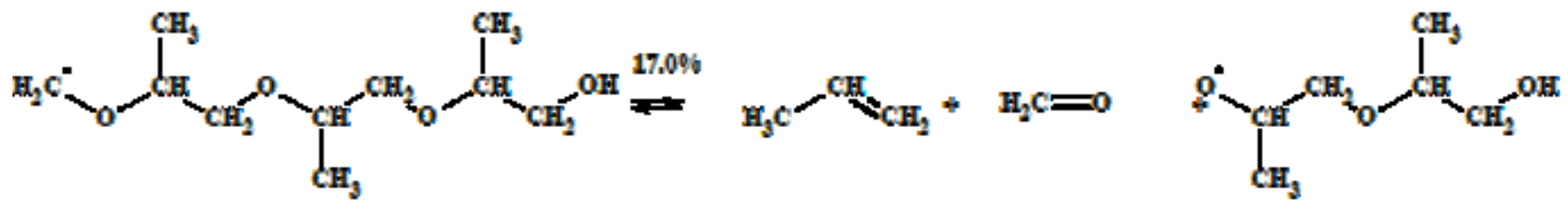

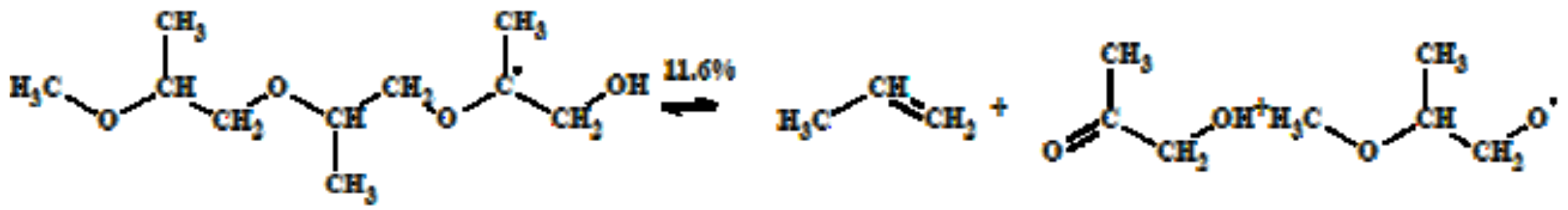

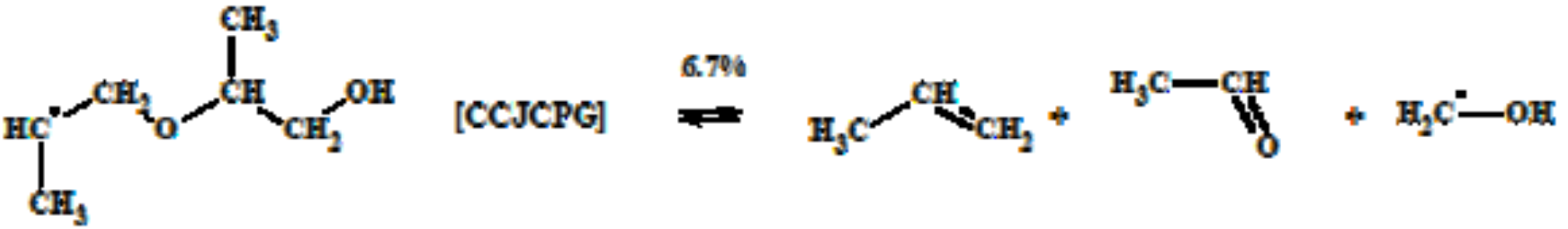




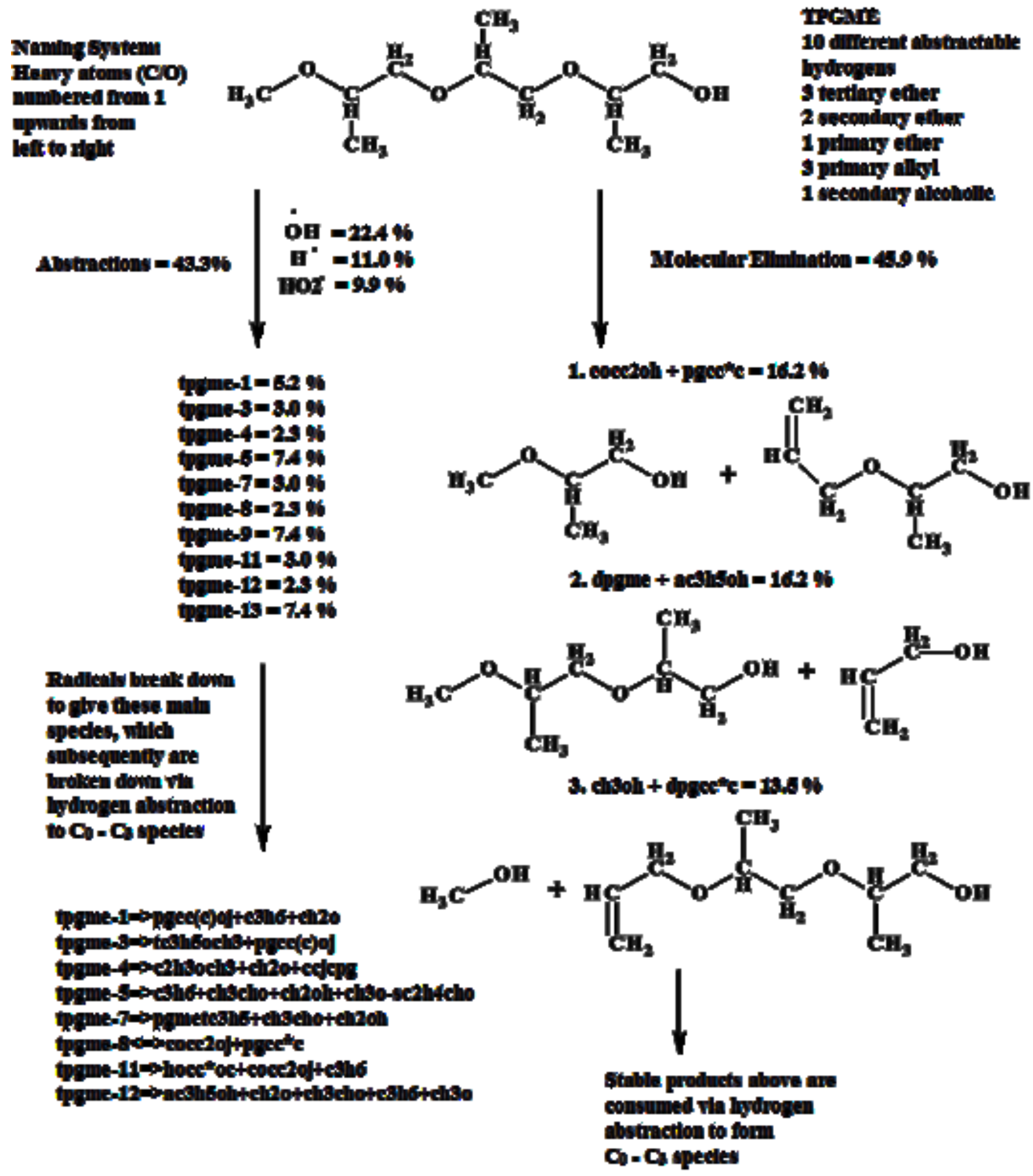

Full structures of nadleal specles above is proviled in the supplentental matedal 


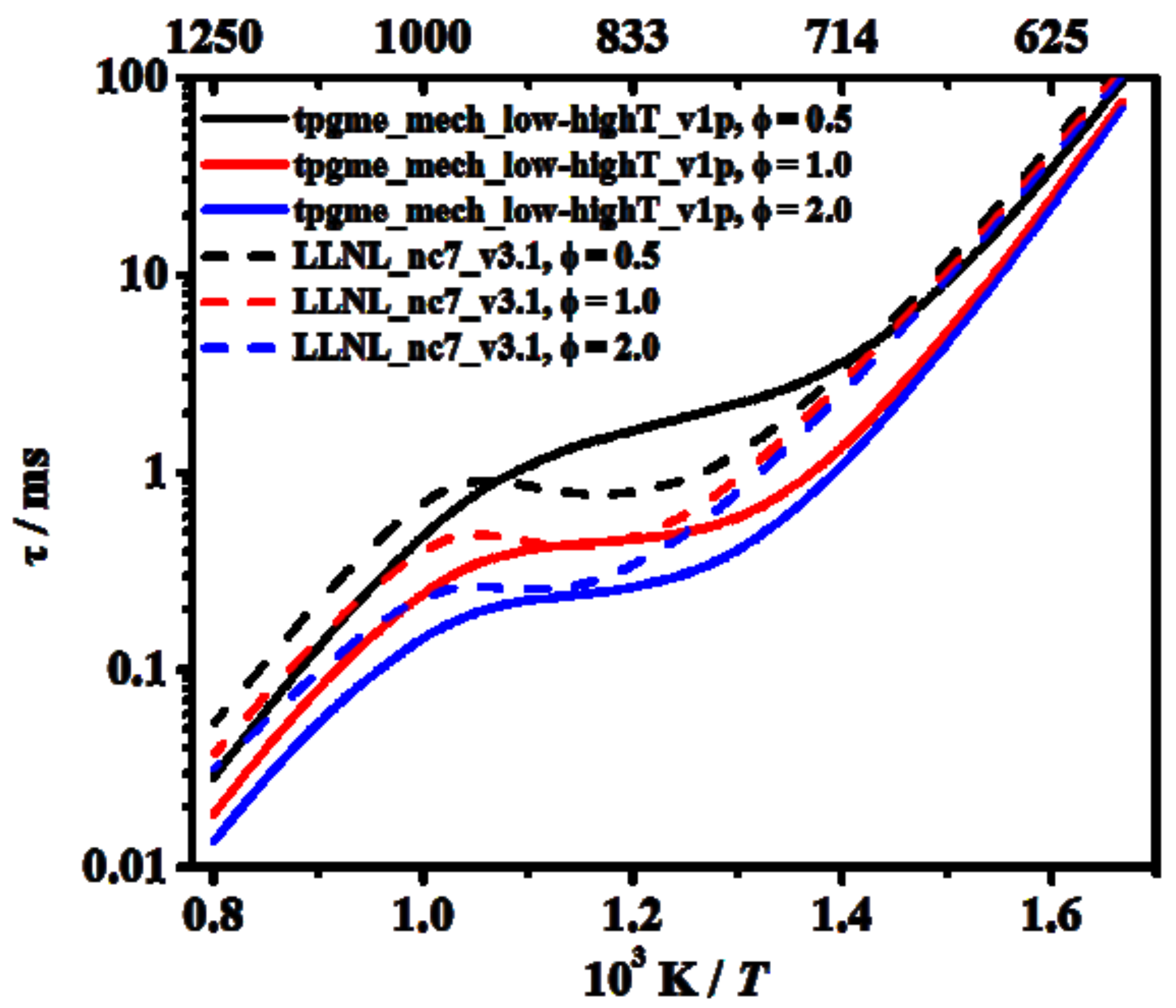




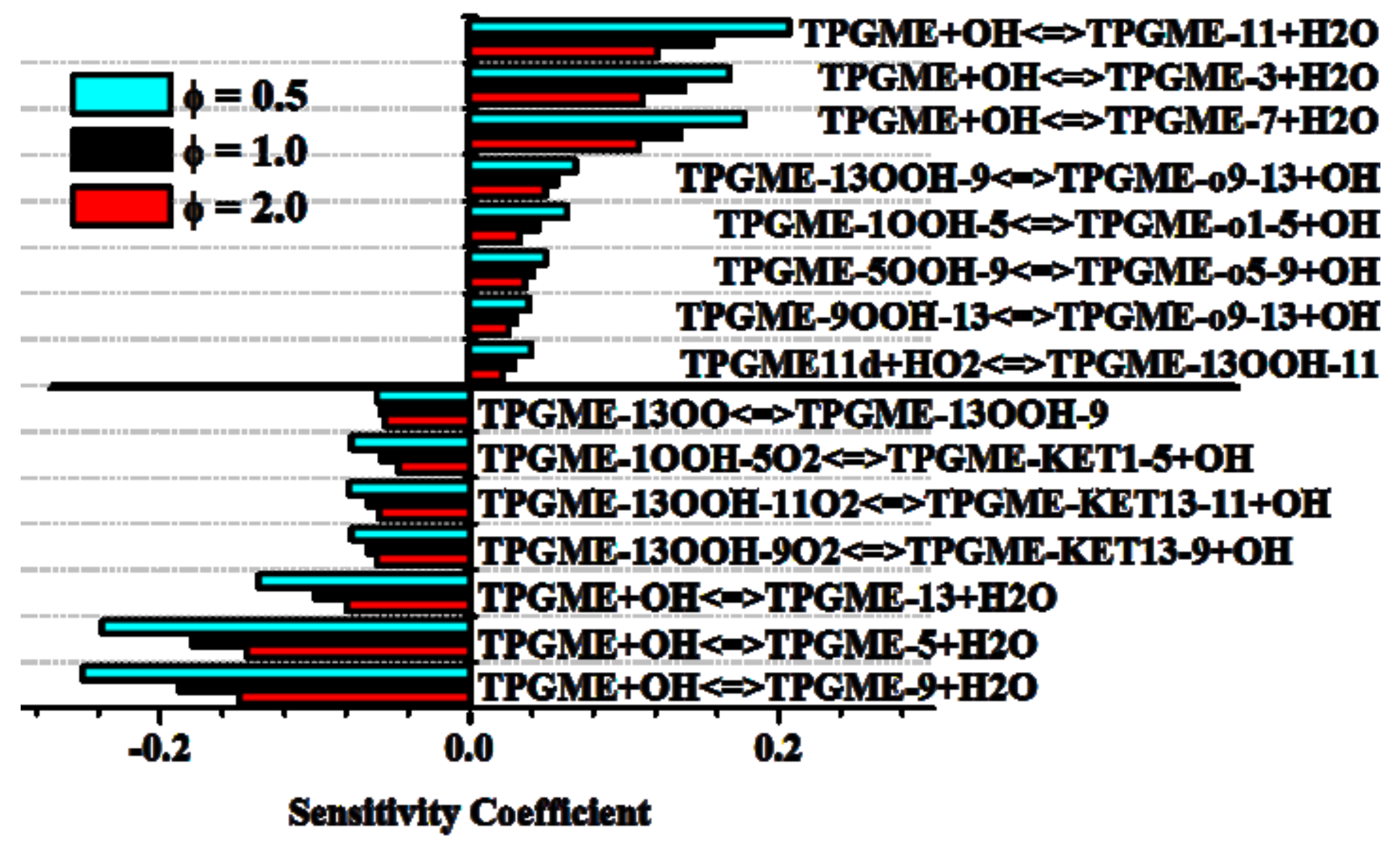




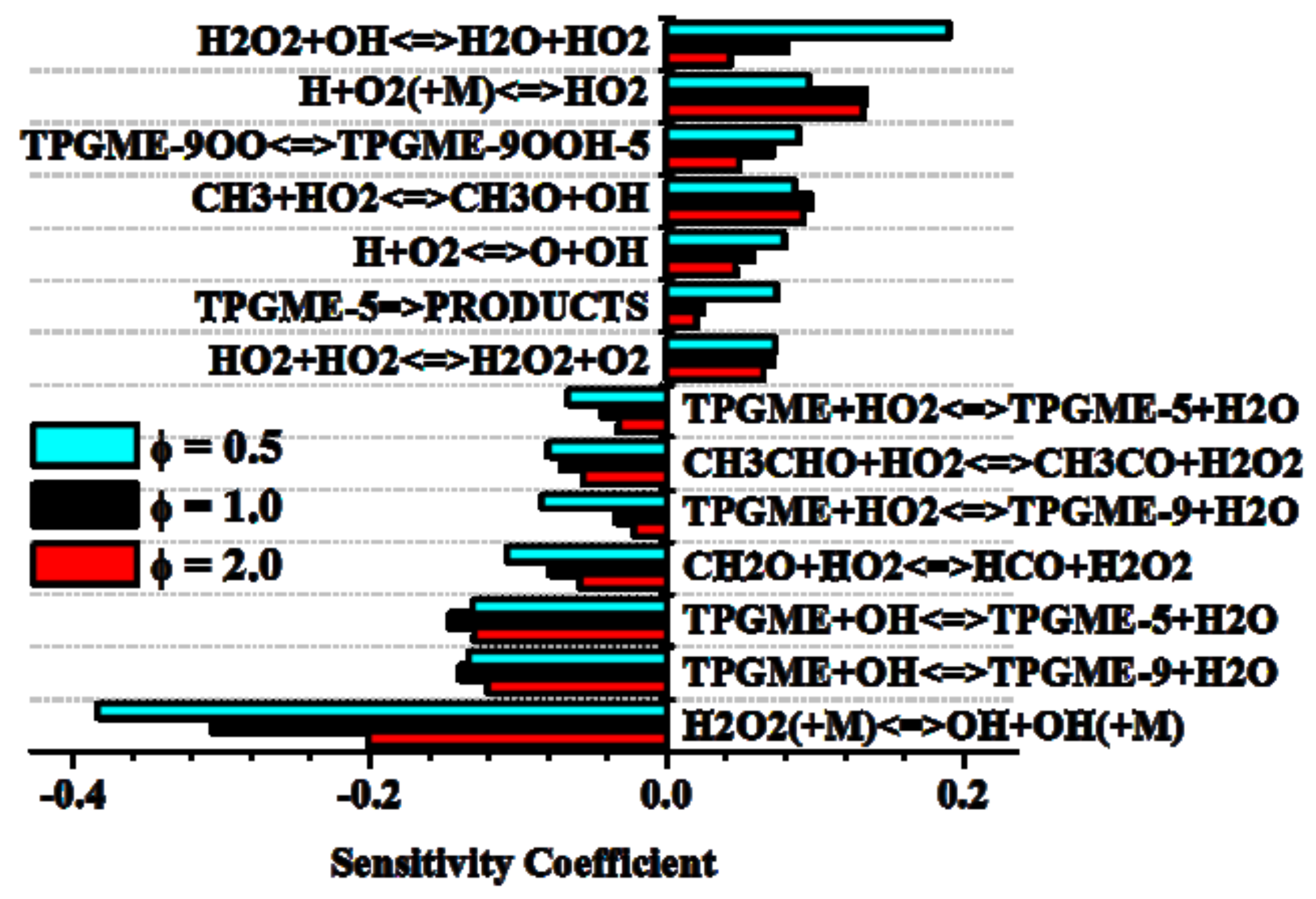

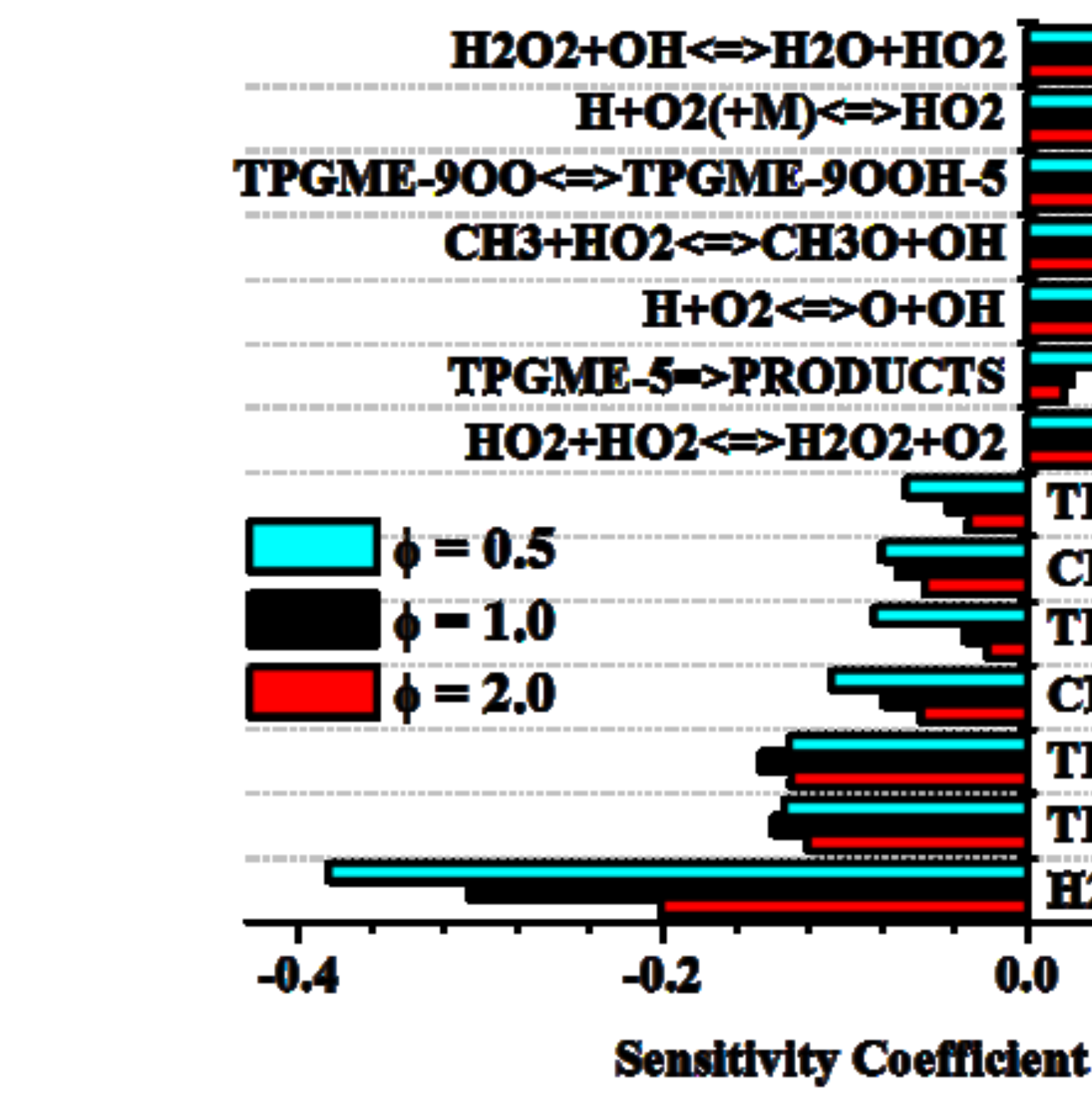

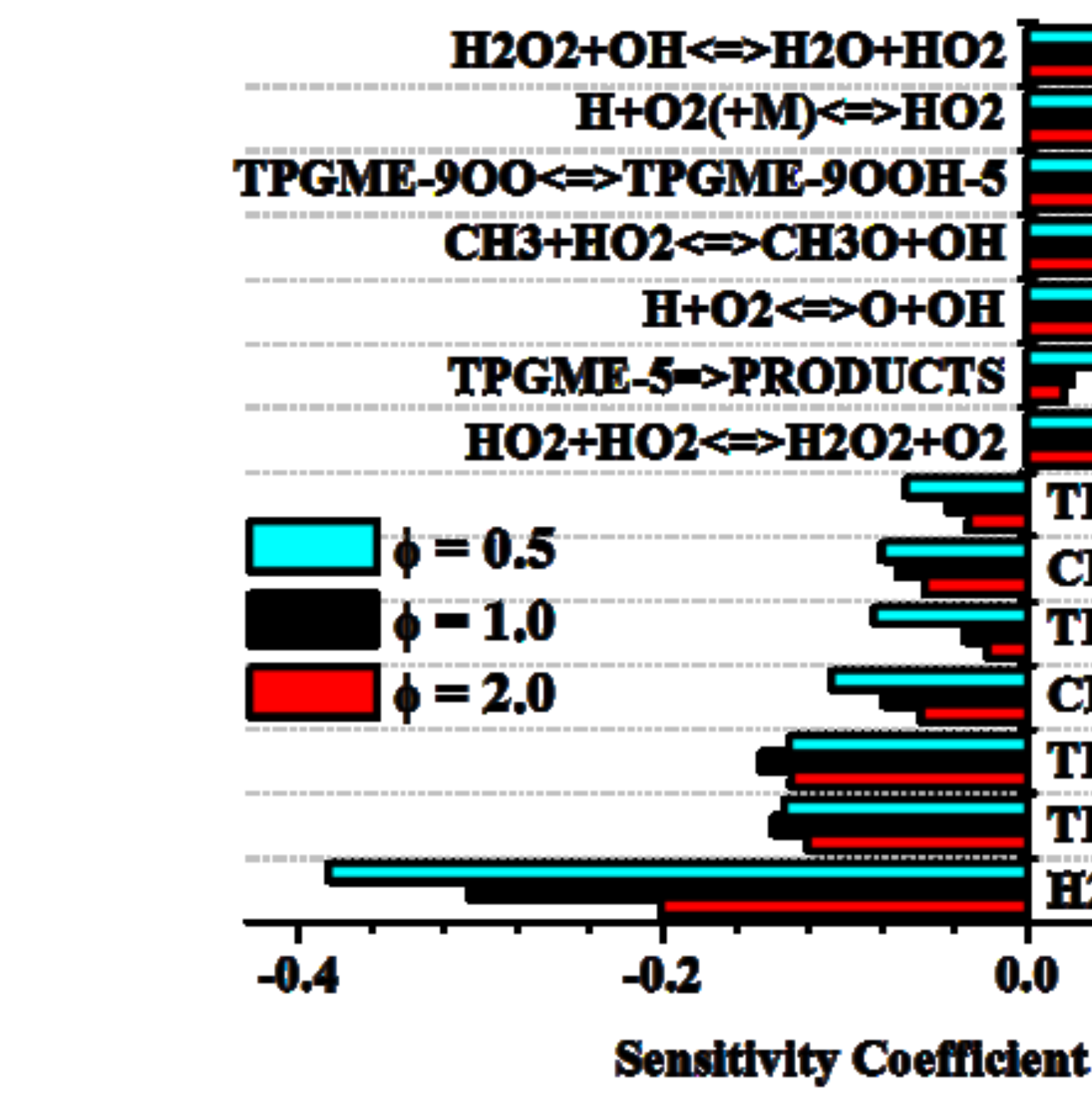

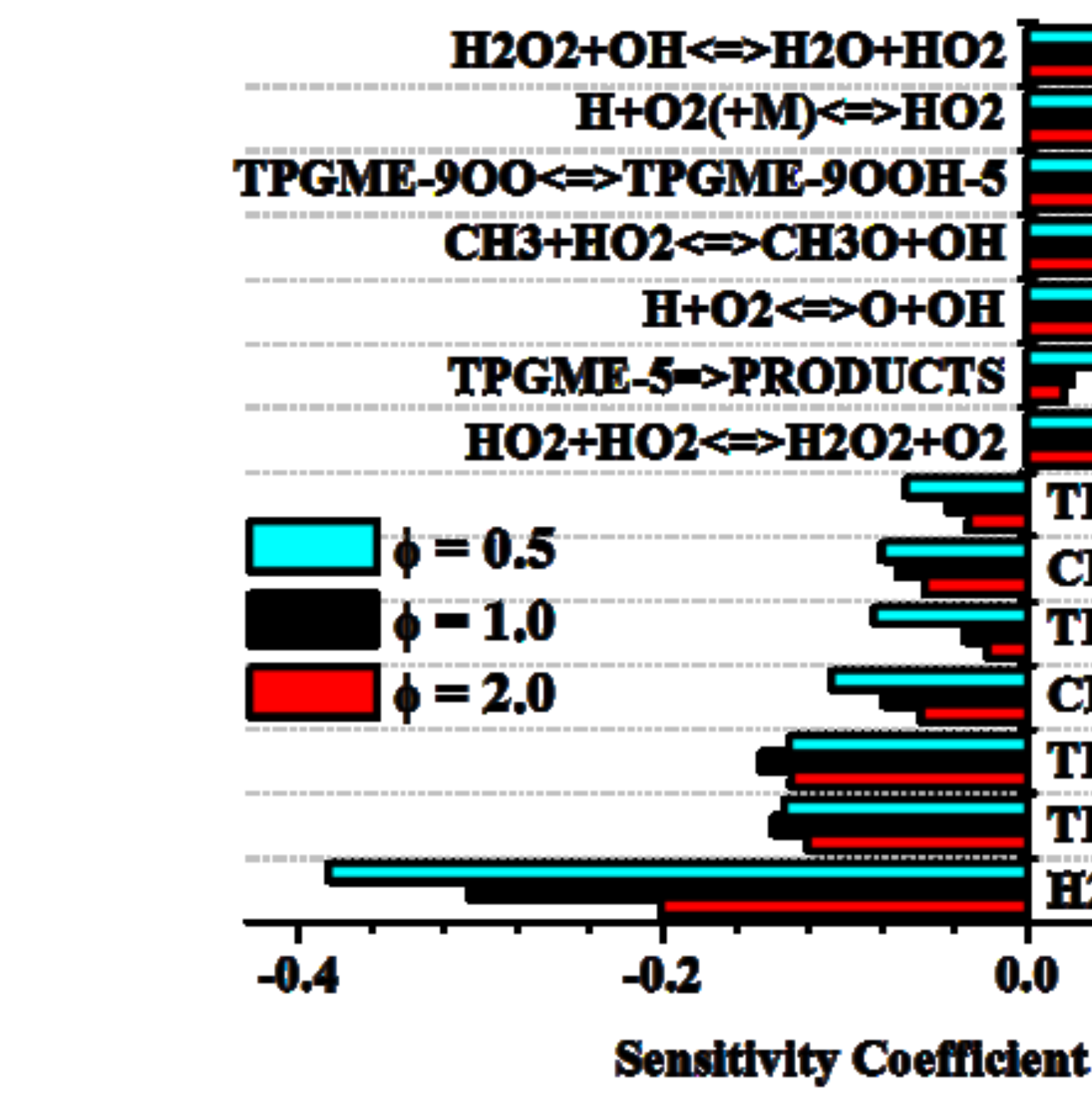

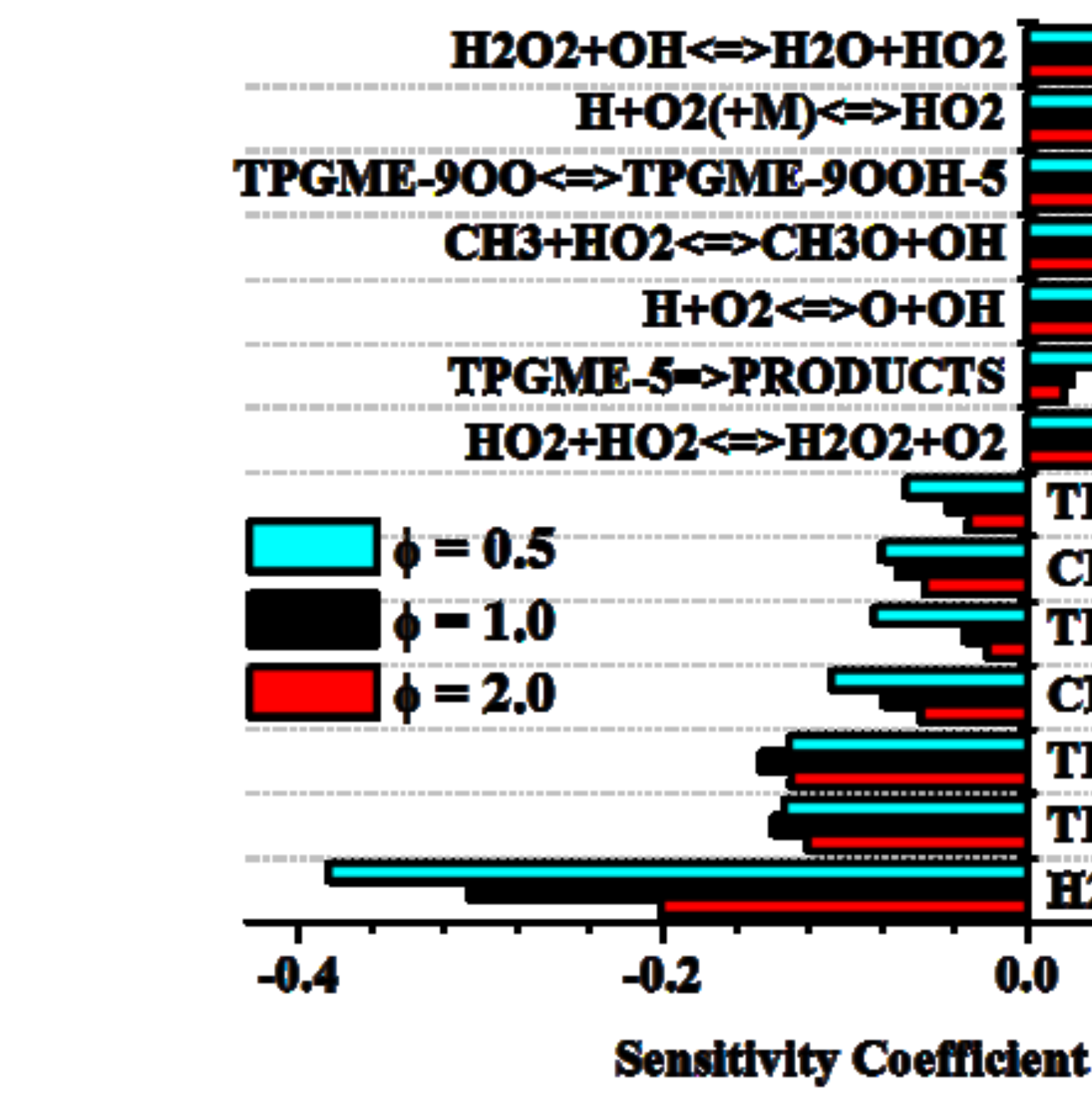

TPGME+HO2 $\Leftrightarrow$ TPGME-5+H2O $\mathrm{CH} 3 \mathrm{CHO}+\mathrm{HO} 2 \Leftrightarrow \mathrm{CH} 3 \mathrm{CO}+\mathrm{H} 2 \mathrm{O} 2$ TPGME+HO2 $\Leftrightarrow$ TPGME-9+H2O $\mathrm{CH} 2 \mathrm{O}+\mathrm{HO} 2<\mathrm{HCO}+\mathrm{H}_{2} \mathrm{O} 2$ TPGME+OH $\Leftrightarrow$ TPGME-5+H2O TPGME+OH $\Leftrightarrow$ TPGME-9+H2O H2O2 $(+M) \Leftrightarrow$ OH $+O H(+M)$

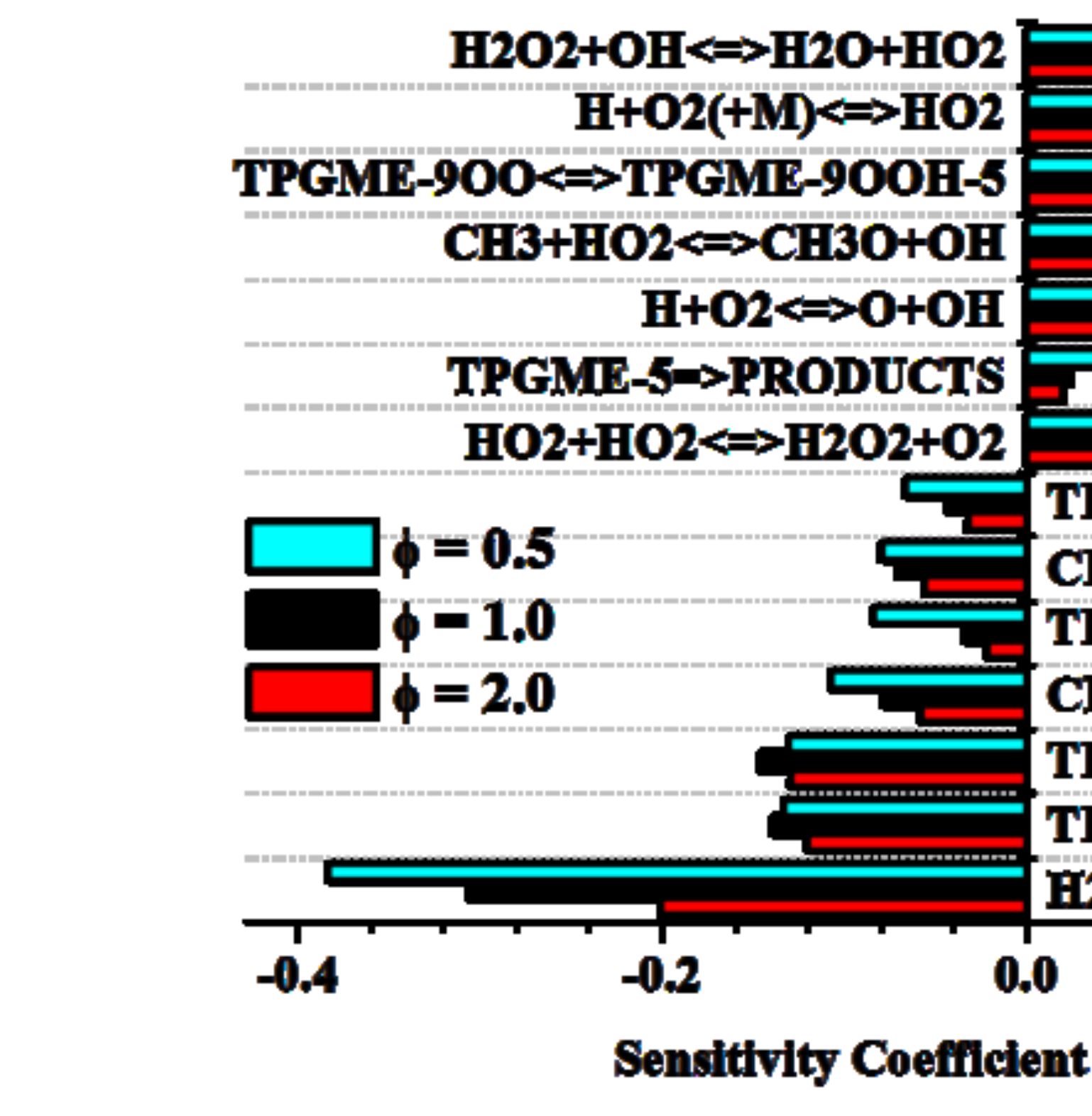

.

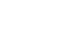

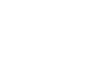

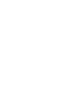

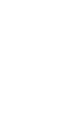




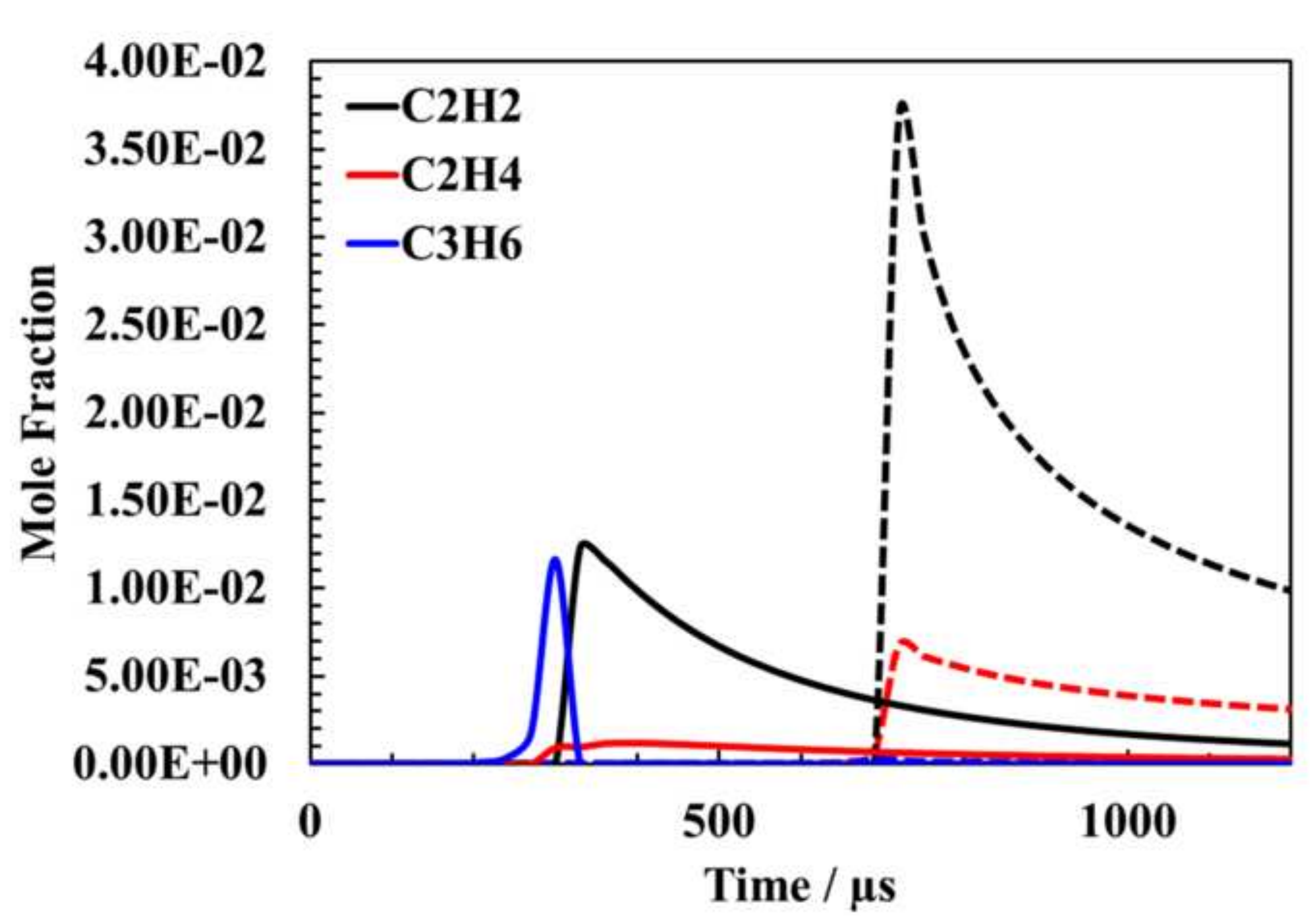




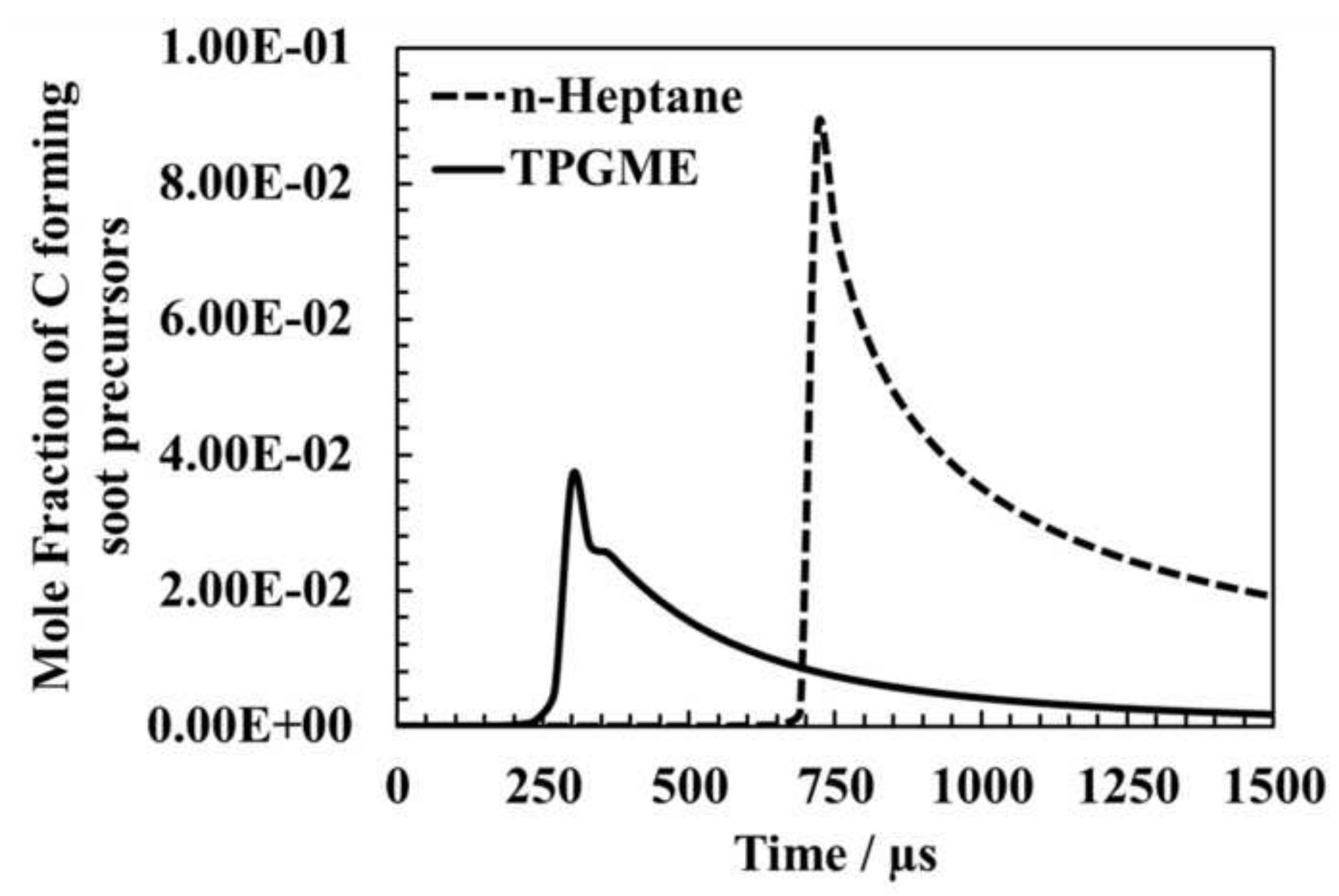

